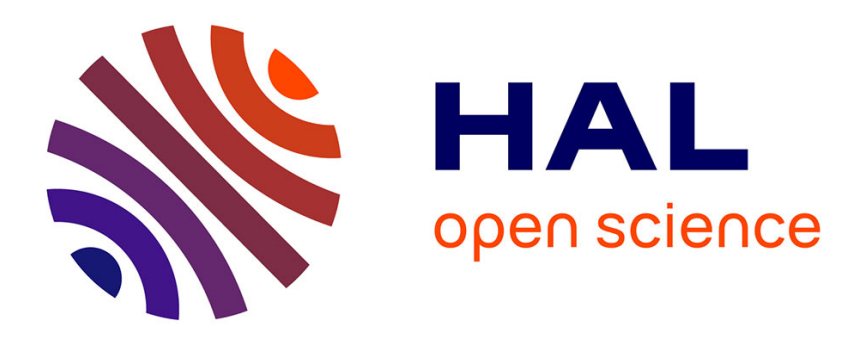

\title{
On four-point penalized Lagrange subdivision schemes
}

X Si, J Baccou, J Liandrat

\section{To cite this version:}

X Si, J Baccou, J Liandrat. On four-point penalized Lagrange subdivision schemes. Applied Mathematics and Computation, 2016, 10.1016/j.amc.2016.01.030 . hal-01265923

\section{HAL Id: hal-01265923 \\ https://hal.science/hal-01265923}

Submitted on 2 Feb 2016

HAL is a multi-disciplinary open access archive for the deposit and dissemination of scientific research documents, whether they are published or not. The documents may come from teaching and research institutions in France or abroad, or from public or private research centers.
L'archive ouverte pluridisciplinaire HAL, est destinée au dépôt et à la diffusion de documents scientifiques de niveau recherche, publiés ou non, émanant des établissements d'enseignement et de recherche français ou étrangers, des laboratoires publics ou privés. 


\title{
On four-point penalized Lagrange subdivision schemes
}

\author{
X. Si ${ }^{\mathrm{a}}$, J. Baccou ${ }^{\mathrm{b}, \mathrm{c}}$, J. Liandrat ${ }^{\mathrm{a}, *}$ \\ ${ }^{a}$ Centrale Marseille, I2M, UMR 7373, CNRS, Aix-Marseille Univ., Marseille, 13453, \\ France \\ ${ }^{b}$ Institut de Radioprotection et de Sûreté Nucléaire(IRSN), PSN-RES, SEMIA, LIMAR, \\ Cadarache, Saint Paul-Lez-Durance, 13115, France \\ ${ }^{c}$ Laboratoire de Micromécanique et d'Intégrité des Structures (MIST), \\ IRSN-CNRS-UMII, B.P. 3, Cadarache, Saint Paul-Lez-Durance, 13115, France
}

\section{Introduction}

Since Deslaurier and Dubuc (Deslauriers and Dubuc (1987)), Lagrange interpolatory subdivision schemes have been intensively used. Originally, they are designed for generating curves starting from initial control polygons by repeated refinements. These schemes and their extensions are working horses for multi-scale approximation and are used in many applications of numerical analysis including numerical solutions of partial differential equations, data reconstruction or image and signal analysis. Their convergence is well understood (at least when they remain linear). Smooth curves can then be generated, leading to efficient tools for approximation problems. Their major drawback lays in the existence of Gibbs oscillations for the limit curve when initial data exhibit strong gradients (see Figure B.4 top left). These oscillations are connected to the oscillations of the coefficients involved in the Lagrange scheme (the set of these coefficients is called the mask of the scheme).

Adaption of the schemes to strong gradients is the motivation of a large family of recently developed schemes: these schemes can be position dependent, as in Baccou and Liandrat (2005) where the monitoring of the position dependency is performed through a segmentation of the data performed a priori, or data dependent (and therefore non-linear) as in Cohen et al. (2003) where the adaption is performed at each application of the scheme, according

\footnotetext{
*Corresponding author, email adress: jliandrat@centrale-marseille.fr, telephone/fax number: 0(33)4-91-05-47-49
} 
to the data. In all these examples, the schemes remain interpolatory. Our goal in this paper, is to introduce a new approach that allows to transform locally an interpolatory scheme into a non interpolatory one, more robust to jump discontinuities. According to the way this approach is applied, it can lead to position dependent schemes (as described in Section 5 of this paper) or to data dependent schemes, as it will be described in a forthcoming publication.

This paper is then devoted to a new approach of Lagrange schemes that allows to transform them locally into non interpolatory ones. The starting point is a recent paper, (Baccou and Liandrat (2013)), where the mask of the Lagrange interpolatory subdivision schemes appeared as the limit mask of a non-stationary subdivision scheme based on Kriging approach. Kriging, (Cressie (1993)), is a stochastic data modeling and reconstruction tool widely used in the framework of spatial data analysis. Its main advantages stand in the possibility to integrate in the reconstruction the spatial dependency of the available data and to quantify the precision of the reconstruction thanks to the model describing this dependency. When they are interpolatory, Kriging schemes are subject to Gibbs oscillations, (Si et al. (2014)), in the vicinity of strong gradients. However, this drawback can be corrected introducing a so called error variance vector, (Chiles and Delfiner (1999)). This vector can be interpreted as a penalization of the oscillations of the subdivision mask coefficients that appear, in the Kriging approach, as solution of an optimization procedure.

The new approach developed in this paper mimics the use of error variance in Kriging to generate new schemes derived from the Lagrange interpolatory framework. They will be called penalized Lagrange subdivision schemes. The paper is organized as follows: after a quick overview of binary subdivision schemes in Section 2, we establish in Section 3 the connection between the Lagrange and Kriging frameworks. It leads to the construction of penalized Lagrange subdivision schemes. This new type of schemes is fully analyzed in Section 4 in the case of a 4-point centered prediction. Then, in Section 5 , it is coupled with a zone-dependent strategy in order to accurately reconstruct discontinuous or locally noisy data. The convergence of this new zone-dependent scheme is studied. Finally, Section 6 provides several numerical tests in order to point out the efficiency of these schemes compared to standard approaches. 


\section{Basic notations and results for binary subdivision schemes}

\subsection{Lagrange interpolatory subdivision scheme}

A (univariate and binary) subdivision scheme $S$ is defined by a linear operator $S: l^{\infty}(\mathbb{Z}) \rightarrow l^{\infty}(\mathbb{Z})$ constructed from a real-valued sequence $\left(a_{k}\right)_{k \in \mathbb{Z}}$ with finite support (called mask of $S$ ) such that

$$
\left(f_{k}\right)_{k \in \mathbb{Z}} \in l^{\infty}(\mathbb{Z}) \mapsto\left((S f)_{k}\right)_{k \in \mathbb{Z}} \in l^{\infty}(\mathbb{Z}) \quad \text { with } \quad(S f)_{k}=\sum_{l \in \mathbb{Z}} a_{k-2 l} f_{l} .
$$

The mask plays a key role in the subdivision process and there exist many works dealing with its construction. Among them, one can mention Dyn (1992), Cohen et al. (2003) and Baccou and Liandrat (2005), that are devoted to interpolatory Lagrange-based schemes. These schemes use masks having $(l+r+1)$ non zero coefficients $\left(a_{0}\right.$ and $\left.a_{2 k+1},-l \leq k \leq r-1\right)$ and interpolatory polynomials of degree $l+r-1$. The sets of non zero coefficients of even index (resp. odd index) of the mask are called the stencils of the scheme. For interpolatory schemes, the even indexed coefficients of the mask are characterized by $a_{0}=1, a_{2 k}=0$ for $k \neq 0$. In the case of Lagrange schemes the odd indexed coefficients of the mask are given by,

$$
a_{2 k+1}=L_{k}^{l, r}\left(-\frac{1}{2}\right),-l \leq k \leq r-1,
$$

where $L_{k}^{l, r}$ is the elementary Lagrange interpolatory polynomial defined by:

$$
L_{k}^{l, r}(x)=\prod_{n=-l, n \neq k}^{r-1} \frac{x-n}{k-n} .
$$

In this article, we will mainly consider the 4-point centered Lagrange interpolatory scheme corresponding to $l=r=2$. It can be written as:

$$
\left\{\begin{array}{l}
(S f)_{2 k}=f_{k}, \\
(S f)_{2 k+1}=-\frac{1}{16} f_{k-1}+\frac{9}{16} f_{k}+\frac{9}{16} f_{k+1}-\frac{1}{16} f_{k+2},
\end{array}\right.
$$

Subdivision is iterated from an initial sequence $\left(f_{k}^{0}\right)_{k \in \mathbb{Z}}$ to generate $\left(f_{k}^{j}\right)_{k \in \mathbb{Z}}$ for $j \geq 1$ as

$$
f^{j+1}=S f^{j}, j \geq 0 .
$$

Parameter $j$ is called the scale parameter and is linked to the dyadic grid $X_{j}=\left\{k 2^{-j}, k \in \mathbb{Z}\right\}$. If the coefficients of the masks are scale invariant, the scheme is stationary whereas it is non-stationary when they depend on $j$. 


\subsection{Convergence of subdivision schemes}

The uniform convergence of subdivision schemes is made precise in the following definition.

\section{Definition 1.}

The subdivision scheme $S$ is said to be uniformly convergent if for any real sequence $\left(f_{k}^{0}\right)_{k \in \mathbb{Z}}$, there exists a continuous function $f$ (called the limit function associated to $\left.f^{0}\right)$ such that: $\forall \epsilon, \exists J$ such that $\forall j \geq J,\left\|S^{j} f^{0}-f(\dot{\dot{2 j}})\right\|_{\infty} \leq \epsilon$.

Following Dyn (1992), the convergence of stationary linear subdivision schemes can be analyzed from the scheme $S_{1}$ associated to the differences $\delta f_{k}^{j}=f_{k+1}^{j}-f_{k}^{j}$ that exists (i.e $\delta f^{j+1}=S_{1} \delta f^{j}$ ) as soon as the initial scheme $S$ reproduces constants, i.e

$$
\forall k \in \mathbb{Z}, f_{k}=1 \Rightarrow \forall k \in \mathbb{Z},(S f)_{k}=1
$$

More precisely, we have:

\section{Theorem 1.}

Let $S$ be a stationary subdivision scheme reproducing constants and $S_{1}$ be the scheme associated to the differences. $S$ is uniformly convergent if and only if $\left(S_{1}\right)^{j} f^{0}$ converges uniformly to the zero function, when $j$ goes to infinity, for all initial data $f^{0}$.

For non-stationary subdivision schemes, the convergence analysis is generally more involved (see Dyn and Levin (1995)). However, the convergence of the mask towards a fixed mask associated to a convergent stationary scheme can be used to reach the result. This will be fully specified in Section 5 .

\section{From ordinary Kriging to penalized Lagrange subdivision}

The main steps related to the definition of a Kriging reconstruction are first recalled in the following section. For sake of clarity, they are described independently of the subdivision framework. 


\subsection{Overview of ordinary Kriging reconstruction}

Considering $N$ observations of a function, $f \in F,\left\{f_{i}\right\}_{i=0, \ldots, N-1}$ on the grid $\left\{x_{i}\right\}_{i=0, . ., N-1}$, Kriging addresses the problem of the reconstruction of $f$ at a new location $x^{\star}$. Ordinary Kriging (Cressie (1993)) belongs to the class of stochastic reconstruction methods. It is assumed that $\left\{f_{i}\right\}_{i \in \mathbb{Z}}$ are realizations of a subset of random variables $\left\{\mathcal{F}\left(x_{i}\right), i \in \mathbb{Z}\right\}$ coming from a random process $\mathcal{F}(x)$ that can be decomposed as $\mathcal{F}(x)=m+\delta(x)$, where $m$ is the constant deterministic mean structure of $\mathcal{F}(x)$ and $\delta(x)$ is a spatially correlated random process. Under stationarity assumptions and constant deterministic mean structure, the spatial structure associated to $\delta(x)$ is identified to the spatial correlation of the data and is exhibited by computing the semi-variogram, $\gamma(h)=\frac{1}{2} E\left((\mathcal{F}(x+h)-\mathcal{F}(x))^{2}\right)$, where $E$ denotes the mathematical expectation ${ }^{1}$. The ordinary Kriging estimator of the random process $\mathcal{F}$ at a new location $x^{\star}$ is denoted $\mathcal{P}\left(\mathcal{F}, x^{\star}\right)$. It is the linear, unbiased predictor $^{2}$ minimizing the estimation variance $\sigma_{K}^{2}=\operatorname{var}\left(\mathcal{F}\left(x^{\star}\right)-\mathcal{P}\left(\mathcal{F}, x^{\star}\right)\right)$. It is written as

$$
\mathcal{P}\left(\mathcal{F}, x^{\star}\right)=\sum_{i=0}^{N-1} \lambda_{i} \mathcal{F}\left(x_{i}\right),
$$

where $\left\{\lambda_{i}\right\}_{i=0, \ldots, N-1}$ are called the Kriging weights. In the case of continuous semi-variograms, expanding the estimation variance as a function of $\gamma$ and taking into account the unbiased condition, the Kriging weights are solutions of the classical constrained optimization problem (Wackernagel (1998)):

$$
\left\{\begin{array}{l}
\min _{\left\{\lambda_{i}\right\}_{i=0, \ldots, N-1}} \mathcal{J}\left(x^{\star} ; \lambda_{0}, \ldots, \lambda_{N-1}\right) \\
\sum_{i=0}^{N-1} \lambda_{i}=1
\end{array}\right.
$$

with $\mathcal{J}\left(x^{\star} ; \lambda_{0}, \ldots, \lambda_{N-1}\right)=-\frac{1}{2} \sum_{i=0}^{N-1} \sum_{n=0}^{N-1} \lambda_{i} \lambda_{n} \gamma\left(\left\|x_{i}-x_{n}\right\|_{2}\right)$ $+\sum_{i=0}^{N-1} \lambda_{i} \gamma\left(\left\|x_{i}-x^{\star}\right\|_{2}\right)$ where $\|.\|_{2}$ stands for the $l^{2}$ Euclidean norm. Denoting by the upper-script $T$ the transpose operator and introducing $\Lambda=$ $\left(\lambda_{0}, \ldots, \lambda_{N-1}\right)^{T}$, Problem (3) leads to the following linear system:

\footnotetext{
${ }^{1}$ Let $X$ be a real random variable of density $f_{X}$. Its mathematical expectation is defined as the integral over the realizations $x$ of $X$ weighted by the density function i.e. $E(X)=\int_{\mathbb{R}} x f_{X}(x) d x$. Moreover, its variance is written as $\operatorname{var}(X)=E\left(X^{2}\right)-(E(X))^{2}$

${ }^{2} \mathcal{P}(\mathcal{F}, x)$ is an unbiased estimator if and only if $E(\mathcal{P}(\mathcal{F}, x)-\mathcal{F}(x))=0$.
} 


$$
\begin{gathered}
{\left[\begin{array}{cc}
R & \mathbb{1} \\
\mathbb{1}^{T} & 0
\end{array}\right]\left[\begin{array}{l}
\Lambda \\
\mu
\end{array}\right]=\left[\begin{array}{l}
b \\
1
\end{array}\right],} \\
\text { where } R=\left[\begin{array}{cccc}
0 & \gamma\left(\left\|x_{0}-x_{1}\right\|_{2}\right) & \ldots & \gamma\left(\left\|x_{0}-x_{N-1}\right\|_{2}\right) \\
\gamma\left(\left\|x_{1}-x_{0}\right\|_{2}\right) & 0 & \ldots & \gamma\left(\left\|x_{1}-x_{N-1}\right\|_{2}\right) \\
\ldots & \ldots & \ldots & 0 \\
\gamma\left(\left\|x_{N-1}-x_{0}\right\|_{2}\right) & \gamma\left(\left\|x_{N-1}-x_{1}\right\|_{2}\right) & \ldots & 0
\end{array}\right], \\
b=\left(\gamma\left(\left\|x_{0}-x^{\star}\right\|_{2}\right), \ldots, \gamma\left(\left\|x_{N-1}-x^{\star}\right\|_{2}\right)\right)^{T} \text { and } \mathbb{1}=(1, \ldots, 1)^{T} \text {. Here, } \mu
\end{gathered}
$$

is the Lagrange multiplier enforcing the unbiasedness of the estimator.

With these notations, the minimization problem (3) can be reformulated as:

$$
\left\{\begin{array}{l}
\min _{\Lambda \in \mathbb{R}^{N}}\left(-\frac{1}{2}<R \Lambda, \Lambda>_{2}+<b, \Lambda>_{2}\right) \\
<\Lambda, \mathbb{1}>_{2}=1
\end{array}\right.
$$

where $<,>_{2}$ is the Euclidean scalar product. The matrix $R$ is symmetric, Toeplitz if the grid $\left\{x_{i}\right\}_{i=0, . ., N-1}$ is regular, with positive coefficients and has a null main diagonal. By definition (Wackernagel (1998)), it satisfies $<R v, v>_{2}<0, \forall v \in \mathbb{R}^{N}$ such that $v \neq 0$ and $\sum_{i=1}^{N} v_{i}=0$. Since any vector $\Lambda$ satisfying $\left\langle\Lambda, \mathbb{1}>_{2}=1\right.$ can be decomposed as $\Lambda=v+\frac{1}{N} \mathbb{1}$ with $<v, \mathbb{1}>_{2}=\sum_{i=1}^{N} v_{i}=0$,

$$
\begin{aligned}
-\frac{1}{2}<R \Lambda, \Lambda>_{2}+<b, \Lambda>_{2}= & -\frac{1}{2}<R v, v>_{2}+<b, v>_{2}-\frac{1}{N}<R v, \mathbb{1}> \\
& -\frac{1}{2 N^{2}} \sum_{i=1}^{N} \sum_{n=1}^{N} R_{i, n}+\frac{1}{N}<b, \mathbb{1}>_{2} .
\end{aligned}
$$

Therefore, using the symmetry of $R$ and introducing $b^{*}=b-\frac{1}{N} R \mathbb{1}$, minimizing $-\frac{1}{2}<R \Lambda, \Lambda>_{2}+<b, \Lambda>_{2}$ under the constraint $<\Lambda, \mathbb{1}>_{2}=1$ is equivalent to minimize $-\frac{1}{2}<R v, v>_{2}+\left\langle b^{*}, v\right\rangle_{2}$. Since $R$ is negative definite on the convex set $\left\{v \in \mathbb{R}^{N}, \sum_{i=1}^{N} v_{i}=0\right\}$ the minimum exists and is unique. Therefore the Kriging weights are well defined.

The previous Kriging estimator (2) is an exact interpolator, since the reconstruction at any position $x^{\star}=x_{i}$ is exactly $f_{i}$. The constraint of exact interpolation can be released introducing non-zero coefficients on the diagonal of $R$ i.e. replacing $R$ by $R-C$ where:

$$
C=\left[\begin{array}{cccc}
c_{1} & 0 & \ldots & 0 \\
0 & c_{2} & 0 & 0 \\
\ldots & \ldots & \ldots & \\
0 & 0 & \ldots & c_{N}
\end{array}\right]
$$


with $\forall i, c_{i} \geq 0$. We call

$$
\mathcal{C}=\left(c_{1}, \ldots, c_{N}\right)
$$

the vector of error variance (Chiles and Delfiner (1999)) $(C=\operatorname{diag}(\mathcal{C}=$ $\left.\left.\left(c_{1}, \ldots, c_{N}\right)\right)\right)$ since it allows to take into account measurement errors or uncertainties on the data. As a result, the corresponding prediction is no more interpolating at points where the error variance vector is non-zero. The optimization problem (5) becomes in this case:

$$
\left\{\begin{array}{l}
\min _{\Lambda \in \mathbb{R}^{N}}\left(-\frac{1}{2}<R \Lambda, \Lambda>_{2}+\frac{1}{2}<C \Lambda, \Lambda>_{2}+<b, \Lambda>_{2}\right) \\
<\Lambda, \mathbb{1}>_{2}=1
\end{array}\right.
$$

and the vector $\mathcal{C}$ can be interpreted as a penalization of the $l_{2}$-norm of $\Lambda$, i.e as a penalization of the oscillations of the coefficients of $\Lambda$, keeping in mind that $<\Lambda, \mathbb{1}\rangle_{2}=1$. With the same arguments as previously, the minimum of (8) exists and is unique.

This short review shows that the Kriging approach is flexible enough to include interpolatory and non-interpolatory reconstructions in the same framework. In the next section, this flexibility is adapted to the Lagrange framework by reformulating the Lagrange subdivision scheme as a Kriginglike reconstruction at dyadic points, i.e. by constructing linear systems of type (4) which solution are the Lagrange stencils. Then (Section 4), a penalization vector $\mathcal{C}$ is introduced and a general class of penalized Lagrange schemes is derived and studied.

\subsection{Combining interpolatory and non-interpolatory reconstructions within the Lagrange scheme framework}

The reformulation of the 4-point interpolatory Lagrange subdivision scheme inside the Kriging framework is summarized in the following proposition, recalling that the Lagrange stencils are $\left(-\frac{1}{16}, \frac{9}{16}, \frac{9}{16},-\frac{1}{16}\right)$ and $(0,1,0,0)$ :

\section{Proposition 1.}

For any $\left(b_{0} \neq 0, b_{1} \neq 0\right)$, let us introduce the polynomial $P_{j}(x)=b_{0} 2^{-2 j} x^{2}+$ $b_{1} 2^{-4 j} x^{4}$ and define the matrix 


$$
R=\left[\begin{array}{cccc}
0 & P_{j}(1) & P_{j}(2) & P_{j}(3) \\
P_{j}(1) & 0 & P_{j}(1) & P_{j}(2) \\
P_{j}(2) & P_{j}(1) & 0 & P_{j}(1) \\
P_{j}(3) & P_{j}(2) & P_{j}(1) & 0
\end{array}\right]
$$

as well as the vectors

$b=\left(P_{j}\left(\frac{3}{2}\right), P_{j}\left(\frac{1}{2}\right), P_{j}\left(\frac{1}{2}\right), P_{j}\left(\frac{3}{2}\right)\right)^{T}\left(\right.$ resp. $\left.b=\left(P_{j}(1), P_{j}(0), P_{j}(1), P_{j}(2)\right)^{T}\right)$.

The unique solutions of Equation (4) are $\left(-\frac{1}{16}, \frac{9}{16}, \frac{9}{16},-\frac{1}{16}, \mu_{L}\right)$ (resp. $(0,1,0,0,0))$ where $\mu_{L}=P_{j}\left(\frac{3}{2}\right)-\left(\frac{9}{16} P_{j}(1)+\frac{9}{16} P_{j}(2)-\frac{1}{16} P_{j}(3)\right)$.

\section{Proof:}

The details of the proof are not given in the sequel. The result stated by this proposition can be obtained by performing the same calculation as in the proof of Proposition 4.3 (Step 3) in Baccou and Liandrat (2013) dealing with the asymptotical analysis of Kriging-based subdivision schemes. In this paper, the polynomial $P_{j}$ that appeared was $2^{-2 j} x^{2}-\frac{1}{2} 2^{-4 j} x^{4}$ as the first terms of the Taylor expansion of the Gaussian semi-variogram $\gamma(x)=1-e^{-2^{-2 j} x^{2}}$. The proof was based on two steps: we first verified that the Lagrange stencils are solutions of Equation (4) with $\mu=\mu_{L}$ or $\mu=0$, then proved the uniqueness of the solution. This last point was not straightforward since the polynomial involved in (9) is not a semi-variogram (Wackernagel (1998)) meaning that the stochastic argument classically used to ensure the invertibility of Kriging systems is not valid in this case. However, studying the kernel of the left hand side matrix of (4), one can derive a linear system involving a Vandermonde matrix that leads to the expected result.

\section{Remark 1.}

The main ingredient of the previous proof is the connection between asymptotical Kriging-based subdivision schemes and Lagrange ones. The choice of the polynomials $P_{j}$ in Proposition 1 that generalize the two first terms of the Taylor expansion of a Gaussian semi-variogram is just a consequence of this key point.

As in the previous section, one can now introduce a penalization vector $\mathcal{C}$ of type (7) to modify the diagonal of $R(9)$. Provided the new matrix $R-C$ 
is invertible (this point is addressed in the next section), it is easy to check that the solution of this new system does not lead to an interpolatory scheme but to a new class of penalized Lagrange schemes that are fully specified in the next section.

\section{A new class of penalized Lagrange subdivision schemes}

\subsection{Construction of the schemes}

Starting from (1), the following definition introduces this new type of schemes.

\section{Definition 2.}

Let $\left\{\mathcal{C}_{i}\right\}_{i \in \mathbb{Z}}$ be a set of penalization vectors and assume that the matrices $R-C_{i}(i \in \mathbb{Z})$ are invertible. The penalized Lagrange subdivision scheme associated to $\left\{\mathcal{C}_{i}\right\}_{i \in \mathbb{Z}}$ is then written :

$$
\left\{\begin{array}{l}
(S f)_{2 i}=\sum_{m=0}^{3} \lambda_{m}^{2 i, \mathcal{C}_{i}} f_{i-1+m} \\
(S f)_{2 i+1}=\sum_{m=0}^{3} \lambda_{m}^{2 i+1, \mathcal{C}_{i}} f_{i-1+m},
\end{array}\right.
$$

where $\left\{\lambda_{m}^{2 i, \mathcal{C}_{i}}\right\}_{m \in\{0, \ldots, 3\}}$ (resp. $\left\{\lambda_{m}^{2 i+1, \mathcal{C}_{i}}\right\}_{m \in\{0, \ldots, 3\}}$ ) are the four first components of the unique solutions of Equation (4) with $R$ (given by Equation (9)) is replaced by $R-C_{i}$ and $b=\left(P_{j}\left(\frac{3}{2}\right), P_{j}\left(\frac{1}{2}\right), P_{j}\left(\frac{1}{2}\right), P_{j}\left(\frac{3}{2}\right)\right)^{T}$ (resp. $\left.b=\left(P_{j}(1), P_{j}(0), P_{j}(1), P_{j}(2)\right)^{T}\right)$.

By definition, the choice of the penalization vector plays a key role in the construction of such schemes. Similarly to the Kriging with error variance approach, this quantity can be used to integrate in classical Lagrange schemes extra information on the data. For example, in signal processing or risk analysis studies, it can be associated to noise or uncertainty in the observations and the corresponding subdivision schemes will lead to a prediction more adapted to the data. More generally, the penalized Lagrange approach offers more flexibility in the stencil construction since the choice of the penalization vector allows to switch from interpolatory $\left(\mathcal{C}_{i}\right.$ is the null vector) to non-interpolatory prediction just by recomputing the left-hand-side matrix of the matricial equation satisfied by the stencil. This property can be point-wise controlled by modifying some components of the penalization 
vector. As a result, it allows to affect more importance to a given set of observations. This advantage is exploited in Section 5 (Definition 3) for the prediction of non-regular data.

Remark 2. It is clear that the parameterization of the stencil construction induces some extra costs in the application of the scheme. Such extra costs also appear in classical position dependent (Baccou and Liandrat (2005)) or non-linear(Cohen et al. (2003)) schemes.

According to Definition 2, the existence of penalized Lagrange stencils relies on the invertibility of the matrices $R-C_{i}, i \in Z Z$ which is fully studied in the next section.

\subsection{Existence and expression of the stencils}

The following propositions are devoted to the analysis of the linear system (4) written with $R-C$ where $R$ is the matrix (9) and $C$ the matrix (6). For induction argument, this global matrix is denoted $A_{0}$ when $C=\mathbf{0}$, where $\mathbf{0}$ stands for the null matrix, and is then used to provide the existence and the expression of the stencils associated to the penalized Lagrange scheme when $C \neq \mathbf{0}$.

\section{Proposition 2.}

Denoting the general term of the symmetric matrix $A_{0}^{-1}$ by $\left[A_{0}^{-1}\right]_{\text {in }}=A_{(i-1)(n-1)}^{(0)}, 1 \leq$ $i, n \leq 5$, then

$$
\forall 0 \leq i \leq 3
$$

$$
\left\{\begin{array}{l}
A_{i 0}^{(0)}=\frac{2^{4 j}}{b_{1}}\left(\frac{\alpha_{1, i}}{24}+\frac{\alpha_{2, i}}{6}+\frac{11}{24} \alpha_{3, i}\right)-\frac{\alpha_{3, i} b_{0} 2^{6 j}}{48 b_{1}^{2}}, \\
A_{i 1}^{(0)}=\frac{2^{4 j}}{b_{1}}\left(-\frac{\alpha_{1, i}}{8}-\frac{5}{12} \alpha_{2, i}-\frac{3}{4} \alpha_{3, i}\right)+\frac{\alpha_{3, i} b_{0} 2^{6 j}}{16 b_{1}^{2}}, \\
A_{i 2}^{(0)}=\frac{2^{4 j}}{b_{1}}\left(\frac{\alpha_{1, i}}{8}+\frac{1}{3} \alpha_{2, i}+\frac{3}{8} \alpha_{3, i}\right)-\frac{\alpha_{3, i} b_{0} 2^{6 j}}{16 b_{1}^{2}}, \\
A_{i 3}^{(0)}=\frac{2^{4 j}}{b_{1}}\left(-\frac{\alpha_{1, i}}{24}-\frac{1}{12} \alpha_{2, i}-\frac{1}{12} \alpha_{3, i}\right)+\frac{\alpha_{3, i} b_{0} 2^{6 j}}{48 b_{1}^{2}},
\end{array}\right.
$$


with

$$
\left[\begin{array}{cccc}
\alpha_{0,0} & \alpha_{0,1} & \alpha_{0,2} & \alpha_{0,3} \\
\alpha_{1,0} & \alpha_{1,1} & \alpha_{1,2} & \alpha_{1,3} \\
\alpha_{2,0} & \alpha_{2,1} & \alpha_{2,2} & \alpha_{2,3} \\
\alpha_{3,0} & \alpha_{3,1} & \alpha_{3,2} & \alpha_{3,3}
\end{array}\right]=\left[\begin{array}{cccc}
1 & 0 & 0 & 0 \\
-\frac{11}{6} & 3 & -\frac{3}{2} & \frac{1}{3} \\
1 & -\frac{5}{2} & 2 & -\frac{1}{2} \\
-\frac{1}{6} & \frac{1}{2} & -\frac{1}{2} & \frac{1}{6}
\end{array}\right]
$$

\section{Proof:}

In this proof, we show how to derive the first equation of System (11). The other equations can be obtained following the same track.

The first column of $A_{0}^{-1},\left(A_{00}^{(0)}, A_{10}^{(0)}, A_{20}^{(0)}, A_{30}^{(0)}, A_{40}^{(0)}\right)^{T}$, is the solution of

$$
A_{0}\left(A_{00}^{(0)}, A_{10}^{(0)}, A_{20}^{(0)}, A_{30}^{(0)}, A_{40}^{(0)}\right)^{T}=(1,0,0,0,0)^{T}
$$

Introducing the polynomial $K(x)=A_{00}^{(0)} P_{j}(x)+A_{10}^{(0)} P_{j}(x-1)+A_{20}^{(0)} P_{j}(x-$ $2)+A_{30}^{(0)} P_{j}(x-3)+A_{40}^{(0)}-L_{0}^{0,4}(x)$, (12) leads to $\forall i \in\{0, \ldots, 3\}, K(i)=0$. Therefore $K$ has 4 roots. Since $A_{00}^{(0)}+A_{10}^{(0)}+A_{20}^{(0)}+A_{30}^{(0)}=0$ (last equation of (12)), $K$ is a polynomial of degree 3 , and finally $\forall x \in \mathbb{R}, K(x)=0$. It implies that the coefficients associated to each power of $x$ are equal to 0 , i.e.

$$
\left\{\begin{array}{l}
\text { for } x^{3}:-4 b_{1} 2^{-4 j} \sum_{i=0}^{3} i A_{i 0}^{(0)}+\frac{1}{6}=0, \\
\text { for } x^{2}: 6 b_{1} 2^{-4 j} \sum_{i=0}^{3} i^{2} A_{i 0}^{(0)}-1=0, \\
\text { for } x:-4 b_{1} 2^{-4 j} \sum_{i=0}^{3} i^{3} A_{i 0}^{(0)}+\frac{11}{6}-\frac{2^{2 j} b_{0}}{12 b_{1}}=0, \\
\text { for } 1: 2^{-2 j} b_{0}\left(A_{10}^{(0)}+4 A_{20}^{(0)}+9 A_{30}^{(0)}\right)+2^{-4 j} b_{1}\left(A_{10}^{(0)}+16 A_{20}^{(0)}+81 A_{30}^{(0)}\right)+A_{40}^{(0)}-1=0,
\end{array}\right.
$$

leading to

$$
\left[\begin{array}{cccc}
1 & 1 & 1 & 1 \\
0 & 1 & 2 & 3 \\
0 & 1 & 4 & 9 \\
0 & 1 & 8 & 27
\end{array}\right]\left[\begin{array}{c}
A_{00}^{(0)} \\
A_{10}^{(0)} \\
A_{20}^{(0)} \\
A_{30}^{(0)}
\end{array}\right]=\left[\begin{array}{c}
0 \\
\frac{1}{24 b_{1}} 2^{4 j} \\
\frac{1}{6 b_{1}} 2^{4 j} \\
\frac{11}{24 b_{1}} 2^{4 j}-\frac{b_{0}}{48 b_{1}^{2}} 2^{6 j}
\end{array}\right]
$$


and $A_{40}^{(0)}=1-2^{-2 j} b_{0}\left(A_{10}^{(0)}+4 A_{20}^{(0)}+9 A_{30}^{(0)}\right)-2^{-4 j} b_{1}\left(A_{10}^{(0)}+16 A_{20}^{(0)}+81 A_{30}^{(0)}\right)$. If $M$ stands for the left hand side matrix in Equation (13), our goal is to calculate $M^{-1}$. Since $M$ is a Vandermonde matrix, its transpose $M^{T}$ connects the basis $\left(1, x, x^{2}, x^{3}\right)$ to the basis $\left(L_{0}^{0,4}(x), L_{1}^{0,4}(x), L_{2}^{0,4}(x), L_{3}^{0,4}(x)\right)$. Therefore, writing $\forall i \in\{0, \ldots, 3\}, L_{i}^{0,4}(x)=\alpha_{3, i} x^{3}+\alpha_{2, i} x^{2}+\alpha_{1, i} x+\alpha_{0, i}$, we have

$$
M^{T}\left[\begin{array}{c}
\alpha_{0, i} \\
\alpha_{1, i} \\
\alpha_{2, i} \\
\alpha_{3, i}
\end{array}\right]=\Delta_{i}
$$

where $\Delta_{i}$ is the vector such that $\forall n \in\{1, \ldots, 4\},\left(\Delta_{i}\right)_{n}=\delta_{n}^{i}$. It then comes out that $\left(\alpha_{0, i} \alpha_{1, i} \alpha_{2, i} \alpha_{3, i}\right)^{T}$ is the $i^{\text {th }}$ column of $\left(M^{T}\right)^{-1}$. Therefore, after a short calculus,

$$
M^{-1}=\left[\begin{array}{cccc}
\alpha_{0,0} & \alpha_{0,1} & \alpha_{0,2} & \alpha_{0,3} \\
\alpha_{1,0} & \alpha_{1,1} & \alpha_{1,2} & \alpha_{1,3} \\
\alpha_{2,0} & \alpha_{2,1} & \alpha_{2,2} & \alpha_{2,3} \\
\alpha_{3,0} & \alpha_{3,1} & \alpha_{3,2} & \alpha_{3,3}
\end{array}\right]=\left[\begin{array}{cccc}
1 & 0 & 0 & 0 \\
-\frac{11}{6} & 3 & -\frac{3}{2} & \frac{1}{3} \\
1 & -\frac{5}{2} & 2 & -\frac{1}{2} \\
-\frac{1}{6} & \frac{1}{2} & -\frac{1}{2} & \frac{1}{6}
\end{array}\right]
$$

Coming back to (13), it finally provides the first equation of System (11). That concludes the proof.

The previous proposition is now used to study the existence of the solutions associated to the penalized Lagrange system. The expression of the solution, derived for the reconstruction of $f_{k}^{j},(k, j) \in \mathbb{Z}^{2}$ when $k=2 i$ and $k=2 i+1$ provides the stencils of the penalized Lagrange scheme (10). Obviously, this analysis depends on the number and on the position of non-zero coefficients in the penalization vector. For sake of simplicity and without loss of generality, we focus on four cases corresponding to the following penalization vectors: $\left(c_{1}, 0,0,0\right),\left(c_{1}, c_{2}, 0,0\right),\left(c_{1}, c_{2}, c_{3}, 0\right)$ and $\left(c_{1}, c_{2}, c_{3}, c_{4}\right)$ with $c_{i}>0$. The four left hand side matrices involved in the penalized Lagrange system (4) are written as: 


$$
A_{m}=\left[\begin{array}{ccccc}
-c_{1} & P_{j}(1) & P_{j}(2) & P_{j}(3) & 1 \\
P_{j}(1) & \ldots & P_{j}(1) & P_{j}(2) & 1 \\
P_{j}(2) & P_{j}(1) & -c_{m} & P_{j}(1) & 1 \\
P_{j}(3) & P_{j}(2) & P_{j}(1) & 0 & 1 \\
1 & 1 & 1 & 1 & 0
\end{array}\right], m \in\{1, \ldots, 4\}
$$

and the corresponding solution is written $\Lambda^{(m)}=\left(\lambda_{0}^{(m)}, \lambda_{1}^{(m)}, \lambda_{2}^{(m)}, \lambda_{3}^{(m)}, \mu^{(m)}\right)^{T}$.

As previously, we write the general term $\left[A_{m}^{-1}\right]_{i n}$ of $A_{m}^{-1}$ as $\left[A_{m}^{-1}\right]_{i n}=A_{(i-1)(n-1)}^{(m)}, 1 \leq$ $i, n \leq 5$. In the following propositions, we focus on the existence and the computation of $\left\{\lambda_{i}^{(m)}\right\}_{i=0, \ldots, 3}$ associated to the systems $A_{m} \Lambda^{(m)}=f$ where $f=\left(P_{j}(1), P_{j}(0), P_{j}(1), P_{j}(2), 1\right)^{T}$ and $f=\left(P_{j}\left(\frac{3}{2}\right), P_{j}\left(\frac{1}{2}\right), P_{j}\left(\frac{1}{2}\right), P_{j}\left(\frac{3}{2}\right), 1\right)^{T}$.

\section{Proposition 3.}

For any $j \in \mathbb{Z}$, if $c_{1} \in D_{j, 1}$ where $D_{j, 1}=\left\{c_{1}>0 / c_{1} \neq \frac{1}{\frac{2^{4 j}}{72 b_{1}}+2^{6 j} \frac{b_{0}}{28861^{2}}}\right\}$ then the solutions of the penalized Lagrange system including one non-zero penalization coefficient exist. They satisfy:

- if $k=2 i$ :

$$
\left[\begin{array}{l}
\lambda_{0}^{(1)} \\
\lambda_{1}^{(1)} \\
\lambda_{2}^{(1)} \\
\lambda_{3}^{(1)}
\end{array}\right]=\left[\begin{array}{l}
0 \\
1 \\
0 \\
0
\end{array}\right]
$$

- if $k=2 i+1$ :

$$
\left[\begin{array}{l}
\lambda_{0}^{(1)} \\
\lambda_{1}^{(1)} \\
\lambda_{2}^{(1)} \\
\lambda_{3}^{(1)}
\end{array}\right]=\left[\begin{array}{c}
-\frac{1}{16}-\frac{c_{1} A_{00}^{(0)}}{16\left(1-c_{1} A_{00}^{(0)}\right)} \\
\frac{9}{16}-\frac{c_{1} A_{10}^{(0)}}{16\left(1-c_{1} A_{00}^{(0)}\right)} \\
\frac{9}{16}-\frac{c_{1} A_{20}^{(0)}}{16\left(1-c_{1} A_{00}^{(0)}\right)} \\
-\frac{1}{16}-\frac{c_{1} A_{30}^{(0)}}{16\left(1-c_{1} A_{00}^{(0)}\right)}
\end{array}\right],
$$


where $\left\{A_{i 0}^{(0)}\right\}_{i=0, \ldots, 3}$ is given in Proposition 2, System (11).

\section{Proof:}

- Existence of the solutions:

Splitting $A_{1}$ as $A_{1}=A_{0}+\tilde{A}_{1}$ and writing, since $A_{0}^{-1}$ exists, $A_{1}=A_{0}(I+$ $\left.A_{0}^{-1} \tilde{A}_{1}\right)$, the invertibility of $A_{1}$ is ensured as soon as $B_{0}=\left(I+A_{0}^{-1} \tilde{A}_{1}\right)$ is invertible. $B_{0}$ can be expressed as:

$$
B_{0}=\left[\begin{array}{ccccc}
1-c_{1} A_{00}^{(0)} & 0 & 0 & 0 & 0 \\
-c_{1} A_{10}^{(0)} & 1 & 0 & 0 & 0 \\
-c_{1} A_{20}^{(0)} & 0 & 1 & 0 & 0 \\
-c_{1} A_{30}^{(0)} & 0 & 0 & 1 & 0 \\
-c_{1} A_{40}^{(0)} & 0 & 0 & 0 & 1
\end{array}\right]
$$

and $\operatorname{det}\left(B_{0}\right)=1-c_{1} A_{00}^{(0)}$. According to the first equation of System (11),

$$
\operatorname{det}\left(B_{0}\right)=1-c_{1}\left(\frac{2^{4 j}}{72 b_{1}}+2^{6 j} \frac{b_{0}}{288 b_{1}^{2}}\right)
$$

It then implies that for any $j \in \mathbb{Z}, B_{0}$ is invertible (and therefore the solutions of the penalized Lagrange system exist) if $c_{1} \neq \frac{1}{\frac{2^{4 j}}{72 b_{1}}+2^{6 j} \frac{b_{0}}{288 b 1^{2}}}$.

- Expression of the solutions:

Using $B_{0}$, the solutions can be evaluated as $\Lambda^{(1)}=B_{0}^{-1} A_{0}^{-1} f$ that, exploiting Proposition (1) and (17), leads to (15) and (16).

The previous proposition can be extended to more than one non-zero penalization constant as follows.

\section{Proposition 4.}

For any $j \in \mathbb{Z}$ and $2 \leq m \leq 4$, let us assume that $c_{1} \in D_{j, 1}$ and denote 
$D_{j, m}=\left\{\left\{c_{k}\right\}_{2 \leq k \leq m} / c_{k}>0, c_{k} \neq \frac{1}{A_{(k-1)(k-1)}^{(k-2)}+\frac{c_{k-1}\left(A_{(k-2)(k-1)}^{(k-2)}\right)^{2}}{1-c_{k-1} A_{(k-2)(k-2)}^{(k-2)}}}\right.$ where for all $1 \leq k \leq m-1,\left\{A_{i n}^{(k)}\right\}_{(i, n) \in\{0, \ldots, 3\}^{2}}$ satisfy the following recursive relation

$$
A_{i n}^{(k)}=A_{i n}^{(k-1)}+\frac{c_{k} A_{i(k-1)}^{(k-1)} A_{(k-1) n}^{(k-1)}}{1-c_{k} A_{(k-1)(k-1)}^{(k-1)}}
$$

If $\left\{c_{k}\right\}_{2 \leq k \leq m} \in D_{j, m}$, the solutions of the penalized Lagrange system including $m$ non-zero penalization coefficients exist. They can also be expressed recursively as:

$$
\forall 2 \leq k \leq m, \lambda_{i}^{(k)}=\lambda_{i}^{(k-1)}+\frac{c_{k} A_{i(k-1)}^{(k-1)}}{1-c_{k} A_{(k-1)(k-1)}^{(k-1)}} \lambda_{k-1}^{(k-1)}
$$

\section{Proof:}

It is performed by induction.

When $m=2$, the proof is similar to the previous one taking into account that $A_{1}$ is invertible (Proposition 3). More precisely, splitting $A_{2}$ as $A_{2}=$ $A_{1}+\tilde{A}_{2}$, the invertibility of $A_{2}$ is connected to the invertibility of $B_{1}=$ $\left(I+A_{1}^{-1} \tilde{A}_{2}\right)$. According to the previous proof, $A_{1}^{-1}=B_{0}^{-1} A_{0}^{-1}$ with $B_{0}$ given by (17). It is then straightforward that $\left\{A_{i n}^{(1)}\right\}_{(i, n) \in\{0, \ldots, 3\}^{2}}$ satisfies (19) with $k=1$. Since $B_{1}$ can be written:

$$
B_{1}=\left[\begin{array}{ccccc}
1 & -c_{2} A_{01}^{(1)} & 0 & 0 & 0 \\
0 & 1-c_{2} A_{11}^{(1)} & 0 & 0 & 0 \\
0 & -c_{2} A_{21}^{(1)} & 1 & 0 & 0 \\
0 & -c_{2} A_{31}^{(1)} & 0 & 1 & 0 \\
0 & -c_{2} A_{41}^{(1)} & 0 & 0 & 1
\end{array}\right]
$$

one can deduce that $\operatorname{det}\left(B_{1}\right)=1-c_{2} A_{11}^{(1)}$. It then comes out that for any $j \in \mathbb{Z}$ and any $c_{1} \in D_{j, 1}, B_{1}$ is invertible (and therefore the solutions of 
the penalized Lagrange problem exist) if $c_{2} \neq \frac{1}{A_{11}^{(0)}+\frac{c_{1}\left(A_{01}^{(0)}\right)^{2}}{1-c_{1} A_{00}^{(0)}}}$. Finally, noticing that

$$
B_{1}^{-1}=\left[\begin{array}{ccccc}
1 & \frac{c_{2} A_{01}^{(1)}}{1-c_{2} A_{11}^{(1)}} & 0 & 0 & 0 \\
0 & 1+\frac{c_{2} A_{11}^{(1)}}{1-c_{2} A_{11}^{(1)}} & 0 & 0 & 0 \\
0 & \frac{c_{2} A_{21}^{(1)}}{1-c_{2} A_{11}^{(1)}} & 1 & 0 & 0 \\
0 & \frac{c_{2} A_{31}^{(1)}}{1-c_{2} A_{11}^{(1)}} & 0 & 1 & 0 \\
0 & \frac{c_{2} A_{41}^{(1)}}{1-c_{2} A_{11}^{(1)}} & 0 & 0 & 1
\end{array}\right]
$$

and since $\Lambda^{(2)}=B_{1}^{-1} A_{1}^{-1} f,(22)$ leads to (20).

Assuming that the solutions of the penalized Lagrange problem including $m$ non-zero coefficients exist and satisfy (20), it remains to show that it is still true for $(m+1)$ non-zero coefficients. This proof is again similar to the previous and won't be provided in the sequel. It is based on the decomposition of $A_{m+1}$ as $A_{m+1}=A_{m}+\tilde{A}_{m+1}$ exhibiting that the invertibility of $A_{m+1}$ is connected to the invertibility of $B_{m}=\left(I+A_{m}^{-1} \tilde{A}_{m+1}\right)$. Using a straightforward generalization of (21) and (22) to get an expression of $B_{k}$ and $B_{k}^{-1}$ with respect to $\left\{A_{i k}^{(k)}\right\}_{i \in\{0, \ldots, 3\}}, k \geq 1$, one can get the expected result.

\subsection{Asymptotical behavior of the stencils}

Propositions 3 and 4 provide the expression of the stencils for any $j$ and any $\left\{c_{i}\right\}_{i \in\{1, \ldots, 4\}}$.In this section, the limits of these stencils when the scale, $j$, or the penalization intensity, $\|\mathcal{C}\|_{\infty}$, goes to infinity are investigated. The first limit will be useful for the convergence analysis of Section 5 while the second is important, in practice, to choose the values of the penalization vector.

\subsubsection{Limit analysis when $j$ goes to infinity}

The following proposition holds: 


\section{Proposition 5.}

For any $\left.\left(c_{1}, c_{2}, c_{3}, c_{4}\right)\right) \in\left(\mathbb{R}^{+}\right)^{4}$, there exists $j^{*}>0$ such that for all $j \geq j^{*}$, the solutions of the penalized Lagrange system exist. Moreover, when $j \rightarrow$ $+\infty$ their limit are the following asymptotical stencils:

- One non-zero penalization coefficient $(m=1)$ :

$$
\begin{aligned}
& - \text { if } k=2 i:\left(\lambda_{0}^{(1)}, \lambda_{1}^{(1)}, \lambda_{2}^{(1)}, \lambda_{3}^{(1)}\right)=(0,1,0,0), \\
& - \text { if } k=2 i+1:\left(\lambda_{0}^{(1)}, \lambda_{1}^{(1)}, \lambda_{2}^{(1)}, \lambda_{3}^{(1)}\right) \rightarrow_{j \rightarrow+\infty}\left(0, L_{-1}^{1,2}\left(-\frac{1}{2}\right), L_{0}^{1,2}\left(-\frac{1}{2}\right), L_{1}^{1,2}\left(-\frac{1}{2}\right)\right) .
\end{aligned}
$$

- Two non-zero penalization coefficients $(m=2)$ :

$$
\begin{aligned}
& - \text { if } k=2 i:\left(\lambda_{0}^{(2)}, \lambda_{1}^{(2)}, \lambda_{2}^{(2)}, \lambda_{3}^{(2)}\right) \rightarrow_{j \rightarrow+\infty}\left(0,0, L_{0}^{0,2}(-1), L_{1}^{0,2}(-1)\right), \\
& - \text { if } k=2 i+1:\left(\lambda_{0}^{(2)}, \lambda_{1}^{(2)}, \lambda_{2}^{(2)}, \lambda_{3}^{(2)}\right) \rightarrow_{j \rightarrow+\infty}\left(0,0, L_{0}^{0,2}\left(-\frac{1}{2}\right), L_{1}^{0,2}\left(-\frac{1}{2}\right)\right) .
\end{aligned}
$$

- Three non-zero penalization coefficients $(m=3)$ : if $k \in\{2 i, 2 i+1\}$ : $\left(\lambda_{0}^{(3)}, \lambda_{1}^{(3)}, \lambda_{2}^{(3)}, \lambda_{3}^{(3)}\right) \rightarrow_{j \rightarrow+\infty}(0,0,0,1)$.

- Four non-zero penalization coefficients $(m=4)$ : if $k \in\{2 i, 2 i+1\}$ :

$$
\left(\lambda_{0}^{(4)}, \lambda_{1}^{(4)}, \lambda_{2}^{(4)}, \lambda_{3}^{(4)}\right) \rightarrow_{j \rightarrow+\infty}\left(\frac{\Pi_{i=1, i \neq m}^{4} c_{i}}{\sum_{n=1}^{4} \Pi_{i=1, i \neq n}^{4} c_{i}}\right)_{1 \leq m \leq 4}
$$

\section{Proof:}

The proof of existence as well as the expression of the limit stencils for one, two and three non-zero penalization coefficients are based on the expression of $\left\{A_{i n}^{(m)}\right\}_{(m, i, n) \in\{1,2\} \times\{0, \ldots, 3\}^{2}}$ obtained combining (11) with (19). For four non-zero penalization coefficients, the limit of $A_{4}$ is invertible. Therefore, for sake of simplicity, we focus only in the sequel on the cases of one and four non-zero penalization coefficient(s). The interested reader can find the full proof for two and three non-zero penalization coefficients in the Annex.

- One non-zero penalization coefficient:

From (18), it is straightforward that for any $c_{1} \in \mathbb{R}^{+}$, $\operatorname{det}\left(B_{0}\right) \sim_{j \rightarrow+\infty}$ $-2^{6 j} c_{1} \frac{b_{0}}{288 b_{1}^{2}}$. Therefore, for large enough $j, B_{0}$ is invertible and the solution exists. 
Moreover, according to (11), we get:

$$
\left\{\begin{array}{l}
A_{10}^{(0)}=-\frac{1}{16 b_{1}} 2^{4 j}-\frac{b_{0}}{96 b_{1}^{2}} 2^{6 j} \\
A_{20}^{(0)}=\frac{1}{24 b_{1}} 2^{4 j}+\frac{b_{0}}{96 b_{1}^{2}} 2^{6 j} \\
A_{30}^{(0)}=\frac{1}{144 b_{1}} 2^{4 j}-\frac{b_{0}}{288 b_{1}^{2}} 2^{6 j}
\end{array}\right.
$$

Plugging the previous expressions in (16) leads to $\lim _{j \rightarrow+\infty}\left(\lambda_{0}^{(1)}, \lambda_{1}^{(1)}, \lambda_{2}^{(1)}, \lambda_{3}^{(1)}\right)=$ $\left(0, \frac{3}{8}, \frac{3}{4},-\frac{1}{8}\right)$, which is the expected expression when $k=2 i+1$.

- Four non-zero penalization coefficients:

Coming back to the linear equation (4), let us first notice that $\lim _{j \rightarrow+\infty} A_{4}=$ $G$ and $\lim _{j \rightarrow+\infty} f=g$ with

$$
G=\left[\begin{array}{ccccc}
-c_{1} & 0 & 0 & 0 & 1 \\
0 & -c_{2} & 0 & 0 & 1 \\
0 & 0 & -c_{3} & 0 & 1 \\
0 & 0 & 0 & -c_{4} & 1 \\
1 & 1 & 1 & 1 & 0
\end{array}\right] \text { and } g=(0,0,0,0,1)^{\prime}
$$

Since $G$ is invertible, $\lim _{j \rightarrow+\infty} \Lambda^{(4)}=G^{-1} g$ and the solution exists. One can easily check that the unique solution of $G U=g$ satisfies $\forall m \in\{1, \ldots, 4\}$, $U_{m}=\frac{\Pi_{i=1, i \neq m}^{4} c_{i}}{\sum_{n=1}^{4} \Pi_{i=1, i \neq n}^{4} c_{i}}$. That concludes the proof.

Following the same track, a similar analysis can be performed to exhibit the limit stencils when the penalization vectors are of types $\left(0, c_{2}, c_{3}, c_{4}\right)$, $\left(0,0, c_{3}, c_{4}\right)$ and $\left(0,0,0, c_{4}\right)$. They are given in Table B.1 and will be used in Section 5 .

\section{[Table 1 about here.]}

The last limit stated by Proposition 5 provides an important result for practical issues. Indeed, for linear subdivision schemes, the variations of sign 
of the coefficients in the stencil are responsible for the oscillations of the limit functions and, as a consequence, of the oscillations of the reconstructed data in the vicinity of strong gradients (Gibbs phenomenon). The constant sign of the coefficients of the limit stencil for four non-zero penalization coefficient scheme implies that, to the limit, the corresponding scheme does not generate the Gibbs oscillations. We refer to Section 6 for an application of this property (Figure B.4, bottom, right).

\subsubsection{Limit analysis when the penalization constants go to infinity}

We now focus on the situation where the penalization vector satisfies $c_{i}=c, 1 \leq i \leq m(m \in\{1, \ldots, 4\})$ and study the limit of the stencils when $c$ goes to infinity.

From Expressions (16) and (20), the limit coefficients depend on $\left\{A_{n m}^{(m)}\right\}_{(m, n) \in\{0, \ldots, 3\}^{2}}$ and therefore possibly on $j$. As a result, for a fully exhaustive description of the limits, many configurations have to be taken into account according to the position and the number of zero coefficients in the previous sequence. For sake of simplicity, in the next proposition, we focus on the case $A_{n m}^{(m)} \neq 0$, $\forall(m, n) \in\{0, \ldots, 3\}^{2}$. This is not restrictive to keep the generality of the results since the subset of scales $I_{n m}^{(m)}=\left\{j \in \mathbb{N}^{+} / A_{n m}^{(m)}=0\right\}$ is finite. Mimicking what has been done when $j \rightarrow+\infty$ and introducing $J^{(m)}=\cup_{n=0}^{3} I_{n m}^{(m)}$, we have:

\section{Proposition 6.}

For any $j \in \mathbb{N}^{+} \backslash \cup_{m=0}^{3} J^{(m)}$ there exists $c^{\star}$ such that for all $c \geq c^{\star}$, the solutions of the penalized Lagrange systems exist. Moreover, when $c \rightarrow+\infty$ their limit are the following:

- One non-zero penalization coefficient:

$$
\begin{aligned}
& \text { - if } k=2 i:\left(\lambda_{0}^{(1)}, \lambda_{1}^{(1)}, \lambda_{2}^{(1)}, \lambda_{3}^{(1)}\right)=(0,1,0,0), \\
& - \text { if } k=2 i+1:\left(\lambda_{0}^{(1)}, \lambda_{1}^{(1)}, \lambda_{2}^{(1)}, \lambda_{3}^{(1)}\right) \rightarrow_{c \rightarrow+\infty} \\
& \quad\left(0, \frac{9}{16}-\frac{3 b_{0} 2^{2 j}+18 b_{1}}{16 b_{0} 2^{2 j}+64 b_{1}}, \frac{9}{16}+\frac{3 b_{0} 2^{2 j}+12 b_{1}}{16 b_{0} 2^{2 j}+64 b_{1}},-\frac{1}{16}+\frac{-b_{0} 2^{2 j}+2 b_{1}}{16 b_{0} 2^{2 j}+64 b_{1}}\right) .
\end{aligned}
$$

- Two non-zero penalization coefficients:

$$
- \text { if } k=2 i:\left(\lambda_{0}^{(2)}, \lambda_{1}^{(2)}, \lambda_{2}^{(2)}, \lambda_{3}^{(2)}\right) \rightarrow_{c \rightarrow+\infty}\left(0,0, \frac{2 b_{0} 2^{2 j}+8 b_{1}}{b_{0} 2^{2 j}+b_{1}},-\frac{b_{0} 2^{2 j}+7 b_{1}}{b_{0} 2^{2 j}+b_{1}}\right),
$$




$$
\begin{aligned}
& - \text { if } k=2 i+1:\left(\lambda_{0}^{(2)}, \lambda_{1}^{(2)}, \lambda_{2}^{(2)}, \lambda_{3}^{(2)}\right) \rightarrow_{c \rightarrow+\infty} \\
& \quad\left(0,0, \lim _{c \rightarrow+\infty} \lambda_{2}^{(1)}+\frac{2 b_{0} 2^{2 j}+8 b_{1}}{b_{0} 2^{2 j}+b_{1}} \lim _{c \rightarrow+\infty} \lambda_{1}^{(1)}, \lim _{c \rightarrow+\infty} \lambda_{3}^{(1)}-\frac{b_{0} 2^{2 j}+7 b_{1}}{b_{0} 2^{2 j}+b_{1}} \lim _{c \rightarrow+\infty} \lambda_{1}^{(1)}\right) .
\end{aligned}
$$

- Three non-zero penalization coefficients: if $k \in\{2 i, 2 i+1\}$ : $\left(\lambda_{0}^{(3)}, \lambda_{1}^{(3)}, \lambda_{2}^{(3)}, \lambda_{3}^{(3)}\right) \rightarrow_{c \rightarrow+\infty}(0,0,0,1)$.

- Four non-zero penalization coefficients: if $k \in\{2 i, 2 i+1\}$ : $\left(\lambda_{0}^{(4)}, \lambda_{1}^{(4)}, \lambda_{2}^{(4)}, \lambda_{3}^{(4)}\right) \rightarrow_{c \rightarrow+\infty}\left(\frac{1}{4}, \frac{1}{4}, \frac{1}{4}, \frac{1}{4}\right)$.

\section{Proof:}

The limit stencils are again evaluated using the limit of $\left\{A_{i n}^{(m)}\right\}_{(m, i, n) \in\{1, \ldots, 3\} \times\{0, \ldots, 3\}^{2}}$ obtained combining (11) with (19). For sake of simplicity, we focus on the case of one penalization coefficient.

From (18), it is straightforward that for any $j \in \mathbb{N}^{+} \backslash \cup_{m=0}^{3} J^{(m)}, \operatorname{det}\left(B_{0}\right) \sim_{c \rightarrow+\infty}$ $-c\left(\frac{2^{4 j}}{72 b_{1}}+2^{6 j} \frac{b_{0}}{288 b 1^{2}}\right)$. Therefore, for large enough $c, B_{0}$ is invertible and the solution exists.

Moreover, plugging the value of $A_{10}^{(0)}, A_{20}^{(0)}, A_{30}^{(0)}$ given by (23) into Expression (16), it comes out

$\lim _{c \rightarrow+\infty}\left(\lambda_{0}^{(1)}, \lambda_{1}^{(1)}, \lambda_{2}^{(1)}, \lambda_{3}^{(1)}\right)=\left(0, \frac{9}{16}-\frac{3 b_{0} 2^{2 j}+18 b_{1}}{16 b_{0} 2^{2 j}+64 b_{1}}, \frac{9}{16}+\frac{3 b_{0} 2^{2 j}+12 b_{1}}{16 b_{0} 2^{2 j}+64 b_{1}},-\frac{1}{16}+\frac{-b_{0} 2^{2 j}+2 b_{1}}{16 b_{0} 2^{2 j}+64 b_{1}}\right)$,

which is the announced expression when $k=2 i+1$.

The corresponding limits for two, three and four non-zero penalization coefficients can be derived following Expression (20).

\subsection{Numerical study}

In this numerical study, the polynomial $P_{j}$ is defined by $P_{j}(x)=100\left(2^{-2 j}\right) x^{2}-$ $2^{-4 j} x^{4}$. Moreover, we still consider a penalization vector characterized by a single coefficient $c$ following our assumption in subsection 4.3.2.

\subsubsection{Critical values of the penalization constant}

Let us first focus on the critical values of $c$ provided by Propositions 3 and 4. These values have to be avoided in order to ensure the existence of the stencils. Table B.2 gives their numerical values for $j \leq 2$ when they are positive. 
[Table 2 about here.]

As expected, the critical values decrease with respect to the scale. Therefore, when $j \geq 1$, the stencils associated to the 4 penalized schemes always exist when $c \in\{0\} \cup[1, \infty[$. This result will be used in Section 6 .

\subsubsection{Stencils dependance on scale and penalization}

We numerically investigate the variations of the stencils according to $j$ and $c$. This study is restricted to the case of a penalization vector with 4 non-zero coefficients that is mainly involved in the zone-dependent subdivision scheme constructed in Section 5.

Figure B.1 displays the stencil coefficients from $j=0$ to $j=10$ when $c=2$.

[Figure 1 about here.]

As expected from Proposition 5, the stencils for large enough values of $j$ converge to $\left(\frac{1}{4}, \frac{1}{4}, \frac{1}{4}, \frac{1}{4}\right)$; moreover, they are of constant positive sign for $j \geq 2$.

Figure B.2 displays the evolution of the stencil coefficients from $c=0$ to $c=100$ when $j=0$. In both plots, the coefficients slowly evolve from the interpolatory Lagrange ones corresponding to $c=0$ towards the limit ones for large $c$. Note that the limit is not yet reached for $c=100$. However, it appears that all the coefficients become non-negative for $c \geq 38$ if $k=2 i$ and for $c \geq 8$ if $k=2 i+1$, a property important to keep in mind when trying to reduce the Gibbs phenomenon.

[Figure 2 about here.]

Table B.3 deals with the limit values of the coefficients. More precisely, it exhibits for each scale the value of $c$ required to obtain the limit $\left(\frac{1}{4}, \frac{1}{4}, \frac{1}{4}, \frac{1}{4}\right)$ up to a $l_{2}$-error bounded by $10^{-3}$. On one hand it shows that for large $j$ $(j \geq 4)$ the coefficients reach their limit values for very small $c$; on the other hand, it points out that for $0 \leq j \leq 3$, there exists a large range of values of $c$ where the coefficients evolve from the interpolatory Lagrange coefficients to the limits.

[Table 3 about here.] 


\section{Zone-dependent penalized Lagrange subdivision scheme}

The penalized Lagrange subdivision schemes are here coupled to a zonedependent strategy in order to derive a new type of subdivision schemes improving the reconstruction of locally non-regular or noisy data. Its definition as well as its convergence analysis are fully specified in what follows.

\subsection{Construction of the scheme}

The scheme is based on the 4-point Lagrange interpolatory scheme associated to a penalization function depending on the position. More precisely:

\section{Definition 3.}

The zone-dependent strategy on the real line $\mathbb{R}$ is characterized by:

- A partition of $\mathbb{R}$ in $p$ zones: $\mathbb{R}=\cup_{0 \leq i \leq p-1} I_{i}$ where $\forall i \leq p-2, I_{i}=$ ]$\left.y_{i}, y_{i+1}\right]$ and $\left.I_{p-1}=\right] y_{p-1}, y_{p}\left[\right.$ with $y_{0}=-\infty, y_{p}=+\infty$ and, for $0 \leq$ $i \leq p-1, y_{i}<y_{i+1}$.

- A penalization function, $\mathcal{P}$, defined piecewise on each interval $I_{i}$ and taking positive values.

For each position $\left(2 i 2^{-(j+1)}\right.$ or $\left.(2 i+1) 2^{-(j+1)}\right)$, the linear system that defines the stencils of the scheme is of type (4) with a left hand side matrix $A_{m}$ given by $(14)$ and $\mathcal{C}=\left(\mathcal{P}\left((i-1) 2^{-j}\right), \mathcal{P}\left(i 2^{-j}\right), \mathcal{P}\left((i+1) 2^{-j}\right), \mathcal{P}\left((i+2) 2^{-j}\right)\right)$.

For sake of clarity and without loss of generality, we focus in the sequel of this section on a three zones partition as $\left.\left.\left.\left.]-\infty, y_{1}\right] \cup\right] y_{1}, y_{2}\right] \cup\right] y_{2},+\infty[$. The penalization function $\mathcal{P}$ is piecewise constant with a non-zero value, $c$, only in the interval $I_{1}$ (Figure B.3).

[Figure 3 about here.]

The reconstruction therefore relies on three different kinds of local predictions based on

- an interpolatory Lagrange subdivision scheme in the interior ${ }^{3}$ of Zones $I_{0}$ and $I_{2}$,

\footnotetext{
${ }^{3}$ If $(l, r)$ characterizes the stencil of the subdivision scheme, the interior of the zone $I$ at level $j$ is the set $I_{e}^{j} \cup I_{o}^{j}$ with $I_{e}^{j}=$ $\left\{x=2 i 2^{-j}\right.$, such that $\exists$ at least $l-1$, resp. $r$, points of $X_{j-1} \cap I$ on the left, resp. right, of $\left.x\right\}$ and $I_{o}^{j}=\left\{x=(2 i+1) 2^{-j}\right.$, such that $\exists$ at least $l$, resp. $r$, points of $X_{j-1} \cap I$ on the left, resp. right, of $\left.x\right\}$
} 
- a penalized Lagrange subdivision scheme with four non-zero penalization coefficients in the interior of $I_{1}$,

- subdivision schemes mixing non-zero penalization vectors of types $(0,0,0,0)$, $(0,0,0, c),(0,0, c, c),(0, c, c, c)$ and $(c, c, c, c)$ in the vicinity of $y_{1}$ and of types $(c, c, c, c),(c, c, c, 0),(c, c, 0,0),(c, 0,0,0)$ and $(0,0,0,0)$ in the vicinity of $y_{2}$.

The resulting scheme is position-dependent (non uniform) and moreover non-stationary since the coefficients of the masks depend on the scale $j$.

\subsection{Convergence analysis}

We prove in this section that the zone-dependent penalized subdivision scheme is convergent. For sake of clarity, the technical details of the proof are given in the Annex and we here focus on the main steps leading to the result.

The key starting point is the following theorem taken from Baccou and Liandrat (2013) that states a sufficient condition of convergence for non stationary (eventually non uniform) schemes.

\section{Theorem 2.}

Let $S$ be a non-stationary subdivision scheme reproducing constants defined by its masks $a_{k}^{j}=\left\{a_{k, m}^{j}\right\}_{m \in \mathbb{Z}},(j, k) \in \mathbb{Z}^{2}$. We suppose that there exists two constants $K<K^{\prime}$, independent of $j$ and $k$ such that $a_{k, m}^{j}=0$ for $m>K^{\prime}$ or $m<K$. If there exists a convergent stationary subdivision scheme $S S$ of masks $a_{k}=\left\{a_{k, m}\right\}_{m \in \mathbb{Z}}, k \in \mathbb{Z}$, with $a_{k, m}=0$ for $m>K^{\prime}$ or $m<K$ and such that

$$
\lim _{j \rightarrow+\infty} \sup _{k \in \mathbb{Z}}\left|a_{k}^{j}-a_{k}\right|=0
$$

then $S$ is convergent.

Remark 3. In Baccou and Liandrat (2013), this theorem addresses the question of the convergence of non-stationary, non-uniform schemes for which there exist a non-uniform stationary asymptotical (i.e when $j \rightarrow+\infty$ ) convergent scheme. The main argument of the proof deals with a contraction property for the scheme for the differences $\left(\delta f_{k}^{j}=f_{k+1}^{j}-f_{k}^{j}\right)$ that is a sufficient condition for convergence of non stationary schemes and a necessary and sufficient condition of convergence for stationary schemes. The uniform 
(with the parameter $k$ ) convergence (with the parameter $j$ ) of the masks indeed ensures that the contraction property is reached for large enough values of $j$.

This result could be compared to the general analysis of convergence for asymptotically equivalent schemes derived in Dyn and Levin (1995). There, with our notations, a sufficient condition for convergence deals with a so called asymptotical equivalence that reads $\sum_{j} \sup _{k \in \mathbb{Z}}\left|a_{k}^{j}-a_{k}\right|$. This condition is a stronger condition than the one used here. However this condition quantifies the distance between the limit functions of the two schemes (non stationary and stationary), a point that is not considered in our result.

Since the asymptotical stencils associated to the penalized Lagrange predictions involved in our zone-dependent scheme have already been identified in Section 4, according to Theorem 2, it is enough to study the convergence of the zone-dependent (non uniform) stationary limit subdivision scheme.

For sake of simplicity and without loss of generality, we suppose that for large enough $j, y_{1}$ ad $y_{2}$ belongs to the dyadic grid $X_{j}$. Precisely, we suppose that:

\section{Definition 4.}

There exists $J>0$ such that for any $j \geq J, y_{1}$ and $y_{2}$ are written respectively as $y_{1}=k_{j, 1} 2^{-j}$ and $y_{2}=k_{j, 2} 2^{-j}$.

Using the previous notations, Table B.4 provides a list of the penalization vectors and of the different stencils associated to the limit scheme according to the position of the point to be predicted.

[Table 4 about here.]

According to Theorem 1, the convergence of the asymptotical scheme is directly connected to the convergence towards 0 of the associated scheme for the differences. Therefore the proof relies on the analysis of the differences $\delta f_{2 i}^{j+1}=f_{2 i+1}^{j+1}-f_{2 i}^{j+1}$ and $\delta f_{2 i+1}^{j+1}=f_{2 i+2}^{j+1}-f_{2 i+1}^{j+1}$ according to their position on the line:

- Interior of $I_{0}$ : $\left\{\delta f_{2 i}^{j+1}\right\}_{0 \leq i \leq k_{j, 1}-2}$ and $\left\{\delta f_{2 i+1}^{j+1}\right\}_{0 \leq i \leq k_{j, 1}-3}$ are computed with the classical Lagrange scheme (i.e. with $(0,0,0,0)$ for the penalization vector). 
- Neighboring intervals around $y_{1}$ (Figure B.9): $\left\{\delta f_{2 i}^{j+1}\right\}_{k_{j, 1}-1 \leq i \leq k_{j, 1}+1}$ and $\left\{\delta f_{2 i+1}^{j+1}\right\}_{k_{j, 1}-2 \leq i \leq k_{j, 1}+1}$ are computed using predictions mixing different penalization vectors (i.e. $(0,0,0,0) /(0,0,0, c)$ to $(0, c, c, c) /(c, c, c, c))$.

- Interior of $I_{1}:\left\{\delta f_{2 i}^{j+1}\right\}_{k_{j, 1}+2 \leq i \leq k_{j, 2}-2}$ and $\left\{\delta f_{2 i+1}^{j+1}\right\}_{k_{j, 1}+2 \leq i \leq k_{j, 2}-3}$ are computed with a prediction associated to the same penalization vector $(c, c, c, c)$.

- Neighboring intervals around $y_{2}:\left\{\delta f_{2 i}^{j+1}\right\}_{k_{j, 2}-1 \leq i \leq k_{j, 2}+1}$ and $\left\{\delta f_{2 i+1}^{j+1}\right\}_{k_{j, 2}-2 \leq i \leq k_{j, 2}+1}$ are computed using predictions mixing different penalization vectors (i.e. $(c, c, c, c) /(c, c, c, 0)$ to $(c, 0,0,0) /(0,0,0,0))$.

- Interior of $I_{2}:\left\{\delta f_{2 i}^{j+1}\right\}_{i \geq k_{j, 2}+2}$ and $\left\{\delta f_{2 i+1}^{j+1}\right\}_{i \geq k_{j, 2}+2}$ are computed with the classical Lagrange scheme (i.e. with $(0,0,0,0)$ for the penalization vector).

It turns out that in the interior zones, the subdivision is performed using a uniform scheme corresponding to the classical 4-point Lagrange interpolatory one in the interior of $I_{0}$ and $I_{2}$ and to the scheme associated to the constant stencil $\left(\frac{1}{4}, \frac{1}{4}, \frac{1}{4}, \frac{1}{4}\right)$ in the interior of $I_{1}$. The convergence analysis in the neighboring intervals around $y_{1}$ and $y_{2}$ is specific due to the non symmetry of the different stencils. Since the convergence property of the classical 4-point Lagrange interpolatory scheme is well known (see for instance Dyn (1992)), the convergence of our scheme is then ensured as soon as the convergence in the interior of $I_{1}$ and, around $y_{1}$ and $y_{2}$ is established. That is precisely what is studied and proved in the Annex.

\section{Numerical applications}

This section provides several numerical tests in order to illustrate the efficiency of the penalized Lagrange subdivision scheme coupled with a zonedependent strategy. In this study, the step of zone detection and choice of the penalization function is decoupled from the prediction one. More precisely, we focus on the capability of our scheme assuming that the zones and the shape of the penalization function are known. The question of the construction of an automatic procedure to achieve the first step is addressed in Remark 5 and will be largely discussed in a forthcoming paper. In the following tests, a special attention is devoted to the analysis of the effect on data reconstruction of the local introduction of a penalization vector. The 
first test is related to the prediction of a discontinuous function while in the second one, noisy data are considered. Again, in all our tests the polynomial model is $P_{j}(x)=100\left(2^{-2 j}\right) x^{2}-2^{-4 j} x^{4}$.

\subsection{Discontinuous data}

In this test, we consider, as initial data $(j=0)$, the sampling of the following step function

$$
f(x)=\left\{\begin{array}{lr}
10 & x \in]-\infty, 8] \\
-10 & x \in] 8,+\infty[
\end{array}\right.
$$

that exhibits a discontinuity at $x=8$.

We first consider the case of a piecewise constant penalization (Figure B.3) analyzed in the previous section, with $\left.\left.I_{1}=\right] y_{1}, y_{2}\right], y_{1}=5$ and $y_{2}=12$. Figure B.4 displays the reconstructed functions starting from a coarse level $j=0$ for different values of the constant $0 \leq c \leq 100$.

[Figure 4 about here.]

As announced, the convergence of the stencils towards constant sign stencils close to the initial discontinuity implies that the Gibbs oscillations disappear for large enough value of the parameter $c$. Moreover, if a too large $c(c=100)$ is chosen, the reconstructed jump can be undesirably smeared out. In this case, the stencil corresponds to the limit one associated to a stationary scheme. However, our non-stationary scheme offers the possibility to reduce this smoothing effect by considering intermediate situations between stationary interpolatory $(c=0)$ and non-interpolatory $(c=100)$ schemes as can be seen on Figure B.4, bottom, left.

\section{Remark 4.}

As illustrated previously, the scheme tends to smooth the discontinuity jump. This is due to the zone-dependent strategy that does not integrate the exact position of the discontinuity as it is classically done when using a positiondependent approach (Baccou and Liandrat (2005)). Therefore, in the framework of reconstruction of discontinuous data, our scheme turns out to be a promising alternative when discontinuity detectors fail and can only provide a discontinuity set of possible discontinuity positions which is often the case in practice due to noise or texture in the data. The penalization function can then be seen as a non-normalized probability density function that controls 
the "likelihood" that a point in the discontinuity set is the exact discontinuity position. The constant function in $I_{1}$ that is considered in this paper implies that any points of the set is likely to be the discontinuity position. However, our scheme allows to affect more importance to a (subset of) point(s) within the set just by switching from the constant function to a piecewise (constant) linear one.

The flexibility of our scheme can also be exploited to work with scale-dependent penalization functions. In this case, the zone $I_{1}$ and the parameter $c$ are redefined at each iteration of the subdivision scheme (they are denoted $I_{1}^{j}$ and $c^{j}$ in the sequel) and a new penalization function is computed. Figure B.5 displays an example of reconstructed step function following this strategy with $I_{1}^{j}=\left[8-\frac{3}{2^{j}}, 8+\frac{4}{2^{j}}\right]$ and $c^{j}=\frac{c^{0}}{2^{4 j}}$. It appears that this adaption of the penalization function reduces the smoothing of the discontinuity jump. This kind of approach leads to non-linear schemes and will be the topic of a forthcoming paper.

[Figure 5 about here.]

\subsection{Locally noisy data}

In real situations, available data can be perturbated by noise. Without specific treatment, the presence of noise strongly deteriorates the reconstruction when interpolatory subdivision schemes are used. In this example, two types of noisy data, related to signal prediction and curve generation, are considered.

\subsubsection{Noisy signal}

The initial data are first generated by sampling the test function $f(x)=$ $0.4 * \sin \left(\frac{x}{3}\right)$ for $x \in \mathbb{R}$. Then a white Gaussian noise (mean 0 , variance 0.4 ) is added in $\left.\left.I_{1}=\right] y_{1}, y_{2}\right]$ with $y_{1}=8$ and $y_{2}=17$.

A zone-dependent penalized Lagrange subdivision scheme with a piecewise constant penalization function is then applied. Figure B.6 exhibits the reconstructed limit functions for different values of $0 \leq c \leq 4000$. Moreover, Table B.5 provides the $l_{2}$-error between the reconstructed sequences at $j=6$ and the sampling of the initial function at the same scale.

It comes out that the introduction of a penalization and its coupling with the zone-dependent strategy lead to a more robust reconstruction in presence of noise. For $c=100$ (Figure B.6, bottom, right), the effect of the noisy data is really damped. This strong improvement is quantified in Table B.5 where 
a reduction of a factor 7 in the $l_{2}$-error between $c=0$ and $c=3726$ can be noticed.

[Figure 6 about here.]

[Table 5 about here.]

\subsubsection{Curve generation}

The same type of zone-dependent penalized Lagrange subdivision scheme is here applied to curve generation. More precisely, we evaluate the capability of this approach in presence of noisy control points. Figure B.7 displays an example of curve reconstruction, where some control points have been polluted by a white noise.

Again, it appears that interpolatory subdivision schemes such as Lagrangebased ones are not tailored to handle noisy data and leads to undesirable oscillations (Figure B.7, left). On the contrary, since the penalized strategy allows to combine interpolatory (in the zone without noise) and noninterpolatory (in the noisy zone) schemes, the oscillations are reduced and the reconstructed curve is more satisfactory (Figure B.7, right).

[Figure 7 about here.]

\section{Remark 5.}

A full penalized Lagrange procedure requires to dsicreminate the different zones in the data and to fix the penalization function (shape and parameters). In some situations, the first point is already performed by previous analysis. It can come for example from a failed discontinuity detection that is not able to provide the exact discontinuity position (see Remark 4) but a set of possible ones. In case of data with uncertainty, it can also be the results of experts' analysis such as in risk studies. However, for an automatical segmentation, a simple way is to proceed by cross-validation (Wackernagel (1998)). More precisely, each observation is removed in turn from the data set and a value at that location is predicted using an interpolatory Lagrange subdivision scheme from the remaining observations. The difference between the data value and the predicted one is then computed in order to provide an indication of the local behavior. The different zones can finally be deduced from the magnitude of this error.

Concerning the choice of the penalization function, it can also be achieved by cross validation using the penalized Lagrange scheme as it is classically 
done in geostatistics to select the semi-variogram model. This procedure has to be performed for each shape and parameter value of the penalization function. Contrarily to the zone detection, the efficiency of each model is not always based on the error between data value and estimated one but has to be defined according to the properties to satisfy. For example, in the case of the prediction of a step function, it can combine both an indicator of the local change in the sign of the derivative for Gibbs oscillations reduction and the $l^{2}$ error to avoid smearing out the jump too much. For noisy data and under an independence assumption of the noise, the efficiency criterion can be based on the estimation of the correlation of the errors between data value and estimated one within $I_{1}$. More precisely, since the scheme is constructed for de-noising, this error can be assimilated to the noise in case of efficient reconstruction and should not be correlated. A (close to) zero correlation therefore indicates an appropriate choice of the penalization function. This procedure can be formalized using statistical tests such as the Durbin-Watson test (Durbin and Watson (1951)).

\section{Conclusion}

A new type of subdivision schemes combining interpolatory and noninterpolatory predictions has been constructed in this article. The starting point is the classical 4-point Lagrange interpolatory scheme. The main ingredient to transform this scheme into an approximating one relies on the connection to Kriging subdivision that offers a more flexible framework to mix interpolatory and non-interpolatory predictions thanks to the introduction of error variances. Plugging the determination of the stencils into that framework allows to define a penalized Lagrange scheme that can be either interpolatory or non-interpolatory according to the choice of the penalization constants. After a full theoretical analysis, this original approach is coupled to a zone-dependent strategy in order to derive non-stationary subdivision schemes adapted to the local behavior of the data to reconstruct. The numerical applications have shown a strong improvement compared to classical Lagrange predictions when data exhibit discontinuities or are perturbated by a noise. 


\section{Appendix A. Existence and expression of the asymptotical sten- cils (Proof of Proposition 5)}

We study for large values of $j$ the quantities $\operatorname{det}\left(B_{1}\right)=1-c_{2} A_{11}^{(1)}$, $\operatorname{det}\left(B_{2}\right)=1-c_{3} A_{22}^{(2)}$ and the coefficients given by (20).

Exploiting the symmetrical property of $A_{1}$ and $A_{2}$, it is therefore enough to derive the asymptotical behavior of the subsets $\left\{A_{01}^{(1)}, A_{02}^{(1)}, A_{03}^{(1)}, A_{11}^{(1)}, A_{12}^{(1)}, A_{13}^{(1)}, A_{22}^{(1)}, A_{23}^{(1)}, A_{33}^{(1)}\right\}$, and $\left\{A_{02}^{(2)}, A_{03}^{(2)}, A_{12}^{(2)}, A_{13}^{(2)}, A_{22}^{(2)}, A_{23}^{(2)}, A_{33}^{(2)}\right\}$.

After a technical calculus one gets

$$
\left\{\begin{array}{l}
A_{01}^{(1)} \sim_{j \rightarrow+\infty} \frac{3}{c_{1}}, \\
A_{02}^{(1)} \sim_{j \rightarrow+\infty}-\frac{3}{c_{1}}, \\
A_{03}^{(1)} \sim_{j \rightarrow+\infty} \frac{1}{c_{1}}, \\
A_{11}^{(1)} \sim_{j \rightarrow+\infty} \frac{1}{24 b_{1}} 2^{4 j}, \\
A_{12}^{(1)} \sim_{j \rightarrow+\infty}-\frac{1}{12 b_{1}} 2^{4 j}, \\
A_{13}^{(1)} \sim_{j \rightarrow+\infty} \frac{1}{24 b_{1}} 2^{4 j}, \\
A_{22}^{(1)} \sim_{j \rightarrow+\infty} \frac{1}{6 b_{1}} 2^{4 j}, \\
A_{23}^{(1)} \sim_{j \rightarrow+\infty}-\frac{1}{12 b_{1}} 2^{4 j}, \\
A_{33}^{(1)} \sim_{j \rightarrow+\infty} \frac{1}{24 b_{1}} 2^{4 j},
\end{array}\right.
$$

and

$$
\left\{\begin{array}{l}
A_{02}^{(2)} \sim_{j \rightarrow+\infty} \frac{3}{c_{1}}, \\
A_{03}^{(2)} \sim_{j \rightarrow+\infty}-\frac{2}{c_{1}}, \\
A_{12}^{(2)} \sim_{j \rightarrow+\infty} \frac{2}{c_{2}}, \\
A_{13}^{(2)} \sim_{j \rightarrow+\infty}-\frac{1}{c_{2}}, \\
A_{22}^{(2)} \sim_{j \rightarrow+\infty}-\frac{1}{2 b_{0}} 2^{2 j}, \\
A_{23}^{(2)} \sim_{j \rightarrow+\infty} \frac{1}{2 b_{0}} 2^{2 j}, \\
A_{33}^{(2)} \sim_{j \rightarrow+\infty}-\frac{1}{2 b_{0}} 2^{2 j},
\end{array}\right.
$$

As a result, it is straightforward that for large enough $j$ the determinants are non-zero, ensuring the existence of the stencils. Moreover, keeping in mind the limit of the stencils in the case of one non-zero penalization coefficient (Proposition 5), one can deduce that 


$$
\begin{aligned}
& \lim _{j \rightarrow+\infty}\left(\lambda_{0}^{(2)}, \lambda_{1}^{(2)}, \lambda_{2}^{(2)}, \lambda_{3}^{(2)}\right)^{T}=\left\{\begin{array}{l}
(0,0,2,-1)^{T} \text { if } k=2 i, \\
\left(0,0, \frac{3}{2},-\frac{1}{2}\right)^{T} \text { if } k=2 i+1,
\end{array}\right. \\
& \text { and } \lim _{j \rightarrow+\infty}\left(\lambda_{0}^{(3)}, \lambda_{1}^{(3)}, \lambda_{2}^{(3)}, \lambda_{3}^{(3)}\right)^{T}=(0,0,0,1)^{T} \text { for any } k \in\{2 i, 2 i+1\},
\end{aligned}
$$

which are the announced results. That concludes the proof.

\section{Appendix B. Proof of the convergence of the stationary subdivi- sion scheme in the interior of $I_{1}$ and, around $y_{1}$ and $y_{2}$ (Section 5.2)}

Interior of $I_{1}$ :

[Figure 8 about here.]

Since $f_{2 i+1}^{j+1}=f_{2 i}^{j+1}=\frac{1}{4}\left(f_{i-1}^{j}+f_{i}^{j}+f_{i+1}^{j}+f_{i+2}^{j}\right)$ and $f_{2 i+2}^{j+1}=\frac{1}{4}\left(f_{i}^{j}+f_{i+1}^{j}+\right.$ $f_{i+2}^{j}+f_{i+3}^{j}$ ) (Figure B.8), It comes that:

$$
\left\{\begin{array}{l}
\delta f_{2 i}^{j+1}=0, \\
\delta f_{2 i+1}^{j+1}=\frac{1}{4}\left[\left(f_{i+3}^{j}-f_{i+2}^{j}\right)+\left(f_{i+2}^{j}-f_{i+1}^{j}\right)+\left(f_{i+1}^{j}-f_{i}^{j}\right)+\left(f_{i}^{j}-f_{i-1}^{j}\right)\right] .
\end{array}\right.
$$

As $\forall j, i, \delta f_{2 i}^{j+1}=0$,

- if $i$ is even $(i=2 m), f_{i+1}^{j}-f_{i}^{j}=f_{2 m+1}^{j}-f_{2 m}^{j}=\delta f_{2 m}^{j}=0$, and $f_{i+3}^{j}-f_{i+2}^{j}=$ $f_{2 m+3}^{j}-f_{2 m+2}^{j}=\delta f_{2 m+2}^{j}=0$. Therefore,

$$
\delta f_{2 i+1}^{j+1}=\frac{1}{4}\left[\left(f_{i+2}^{j}-f_{i+1}^{j}\right)+\left(f_{i}^{j}-f_{i-1}^{j}\right)\right],
$$

- if $i$ is odd $(i=2 m+1), f_{i+2}^{j}-f_{i+1}^{j}=f_{2 m+3}^{j}-f_{2 m+2}^{j}=\delta f_{2 m+2}^{j}=0$, $f_{i}^{j}-f_{i-1}^{j}=f_{2 m+1}^{j}-f_{2 m}^{j}=\delta f_{2 m}^{j}=0$. Therefore,

$$
\delta f_{2 i+1}^{j+1}=\frac{1}{4}\left[\left(f_{i+3}^{j}-f_{i+2}^{j}\right)+\left(f_{i+1}^{j}-f_{i}^{j}\right)\right] .
$$

Since $\left|\frac{1}{4}\right|+\left|\frac{1}{4}\right|<1, \delta f_{2 i+1}^{j+1} \rightarrow_{j \rightarrow+\infty} 0$.

$\underline{\text { Neighboring intervals around } y_{1} \text { : }}$ 
[Figure 9 about here.]

We separate the study for differences involving points on the left and on the right of $y_{1}$ (Figure B.9).

- Left of $y_{1}$

Exploiting Table B.4, the differences can be expressed as follows:

$$
\begin{aligned}
\delta f_{2 k_{j, 1}-3}^{j+1} & =f_{k_{j, 1}-1}^{j}-\left(-\frac{1}{16} f_{k_{j, 1}-3}^{j}+\frac{9}{16} f_{k_{j, 1}-2}^{j}+\frac{9}{16} f_{k_{j, 1}-1}^{j}-\frac{1}{16} f_{k_{j, 1}}^{j}\right) \\
=-\frac{1}{16} \delta f_{k_{j, 1}-3}^{j}+\frac{1}{2} \delta f_{k_{j, 1}-2}^{j}+\frac{1}{16} \delta f_{k_{j, 1}-1}^{j} & \\
\delta f_{2 k_{j, 1}-2}^{j+1} & =\left(-\frac{1}{8} f_{k_{j, 1}-2}^{j}+\frac{3}{4} f_{k_{j, 1}-1}^{j}+\frac{3}{8} f_{k_{j, 1}}^{j}\right)-f_{k_{j, 1}-1}^{j} \\
& =\frac{1}{8} \delta f_{k_{j, 1}-2}^{j}+\frac{3}{8} \delta f_{k_{j, 1}-1}^{j}, \\
\delta f_{2 k_{j, 1}-1}^{j+1} & =f_{k_{j, 1}}^{j}-\left(-\frac{1}{8} f_{k_{j, 1}-2}^{j}+\frac{3}{4} f_{k_{j, 1}-1}^{j}+\frac{3}{8} f_{k_{j, 1}}^{j}\right) \\
& =-\frac{1}{8} \delta f_{k_{j, 1}-2}^{j}+\frac{5}{8} \delta f_{k_{j, 1}-1}^{j}
\end{aligned}
$$

All these differences are contractive since $\left|-\frac{1}{16}\right|+\left|\frac{1}{2}\right|+\left|\frac{1}{16}\right|<1,\left|\frac{1}{8}\right|+\left|\frac{3}{8}\right|<1$ and $\left|-\frac{1}{8}\right|+\left|\frac{5}{8}\right|<1$. Moreover, they only involve differences in $I_{0}$. Combined with the uniform convergence of the classical Lagrange scheme, it therefore implies that $\delta f_{2 k_{j, 1}-3}^{j+1} \rightarrow_{j \rightarrow+\infty} 0, \delta f_{2 k_{j, 1}-2}^{j+1} \rightarrow_{j \rightarrow+\infty} 0$ and $\delta f_{2 k_{j, 1}-1}^{j+1} \rightarrow_{j \rightarrow+\infty} 0$.

- Right of $y_{1}$

They are written as:

$$
\begin{aligned}
\delta f_{2 k_{j, 1}}^{j+1} & =\left(-\frac{1}{2} f_{k_{j, 1}-1}^{j}+\frac{3}{2} f_{k_{j, 1}}^{j}\right)-f_{k_{j, 1}}^{j}, \\
& =\frac{1}{2} \delta f_{k_{j, 1}-1}^{j},
\end{aligned}
$$




$$
\begin{gathered}
\delta f_{2 k_{j, 1}+1}^{j+1}=f_{k_{j, 1}}^{j}-\left(-\frac{1}{2} f_{k_{j, 1}-1}^{j}+\frac{3}{2} f_{k_{j, 1}}^{j}\right) \\
=-\frac{1}{2} \delta f_{k_{j, 1}-1}^{j}, \\
\delta f_{2 k_{j, 1}+2}^{j+1}=0 \\
\delta f_{2 k_{j, 1}+3}^{j+1}=\left(\frac{1}{4} f_{k_{j, 1}+1}^{j}+\frac{1}{4} f_{k_{j, 1}+2}^{j}+\frac{1}{4} f_{k_{j, 1}+3}^{j}+\frac{1}{4} f_{k_{j, 1}+4}^{j}\right)-f_{k_{j, 1}}^{j}, \\
=\delta f_{k_{j, 1}}^{j}+\frac{3}{4} \delta f_{k_{j, 1}+1}^{j}+\frac{1}{2} \delta f_{k_{j, 1}+2}^{j}+\frac{1}{4} \delta f_{k_{j, 1}+3}^{j} .
\end{gathered}
$$

Since the differences (B.1) and (B.2) only depend on differences in $I_{0}$, it is straightforward that $\delta f_{2 k_{j, 1}}^{j+1} \rightarrow_{j \rightarrow+\infty} 0$ and $\delta f_{2 k_{j, 1}+1}^{j+1} \rightarrow_{j \rightarrow+\infty} 0$. Clearly, Expression (B.4) does not imply a contraction from $\delta f^{j}$ to $\delta f^{j+1}$. However, since by Definition $4, \forall j>J, k_{j, 1}=2 k_{j-1,1}$, it can be written as:

$$
\begin{aligned}
\delta f_{2 k_{j, 1}+3}^{j+1}= & \frac{1}{4} \delta f_{2 k_{j-1,1}+3}^{j} \\
& +\delta f_{2 k_{j-1,1}}^{j}+\frac{3}{4} \delta f_{2 k_{j-1,1}+1}^{j}+\frac{1}{2} \delta f_{2 k_{j-1,1}+2}^{j}
\end{aligned}
$$

This expression exhibits a recursive relationship between $\delta f_{2 k_{j, 1}+3}^{j+1}$ and $\delta f_{2 k_{j-1,1}+3}^{j}$ involving the contraction factor $\frac{1}{4}$ and a sum of extra terms that, according to (B.1)-(B.3) converge towards 0. Therefore $\delta f_{2 k_{j, 1}+3}^{j+1} \rightarrow_{j \rightarrow+\infty} 0$.

$\underline{\text { Neighboring intervals around } y_{2} \text { : }}$

Similarly to the previous case, the convergence study is split in two steps. We first focus on the right side of $y_{2}$ then on the left one.

- Right of $y_{2}$

$$
\begin{aligned}
\delta f_{2 k_{j, 2}}^{j+1} & =\left(\frac{3}{2} f_{k_{j, 2}+1}^{j}-\frac{1}{2} f_{k_{j, 2}+2}^{j}\right)-\left(2 f_{k_{j, 2}+1}^{j}-f_{k_{j, 2}+2}^{j}\right) \\
& =\frac{1}{2} \delta f_{k_{j, 2}+1}^{j}
\end{aligned}
$$




$$
\begin{aligned}
\delta f_{2 k_{j, 2}+1}^{j+1} & =f_{k_{j, 2}+1}^{j}-\left(\frac{3}{2} f_{k_{j, 2}+1}^{j}-\frac{1}{2} f_{k_{j, 2}+2}^{j}\right) \\
& =\frac{1}{2} \delta f_{k_{j, 2}+1}^{j} \\
\delta f_{2 k_{j, 2}+2}^{j+1}= & \left(\frac{3}{8} f_{k_{j, 2}+1}^{j}+\frac{3}{4} f_{k_{j, 2}+2}^{j}-\frac{1}{8} f_{k_{j, 2}+3}^{j}\right)-f_{k_{j, 2}+1}^{j}, \\
= & \frac{5}{8} \delta f_{k_{j, 2}+1}^{j}-\frac{1}{8} \delta f_{k_{j, 2}+2}^{j}, \\
\delta f_{2 k_{j, 2}+3}^{j+1}= & f_{k_{j, 2}+2}^{j}-\left(\frac{3}{8} f_{k_{j, 2}+1}^{j}+\frac{3}{4} f_{k_{j, 2}+2}^{j}-\frac{1}{8} f_{k_{j, 2}+3}^{j}\right), \\
= & \frac{3}{8} \delta f_{k_{j, 2}+1}^{j}+\frac{1}{8} \delta f_{k_{j, 2}+2}^{j},
\end{aligned}
$$

All these differences are contractive since $\left|\frac{1}{2}\right|<1,\left|\frac{5}{8}\right|+\left|-\frac{1}{8}\right|<1,\left|\frac{3}{8}\right|+\left|\frac{1}{8}\right|<$ 1. Moreover, they only involve differences in $I_{2}$. Combined with the uniform convergence of the classical Lagrange scheme, it leads to $\delta f_{2 k_{j, 2}}^{j+1} \rightarrow_{j \rightarrow+\infty} 0$, $\delta f_{2 k_{j, 2}+1}^{j+1} \rightarrow_{j \rightarrow+\infty} 0, \delta f_{2 k_{j, 2}+2}^{j+1} \rightarrow_{j \rightarrow+\infty} 0$ and $\delta f_{2 k_{j, 2}+3}^{j+1} \rightarrow_{j \rightarrow+\infty} 0$.

- Left of $y_{2}$

$$
\begin{aligned}
& \delta f_{2 k_{j, 2}-3}^{j+1}= f_{k_{j, 2}+1}^{j}-\left(\frac{1}{4} f_{k_{j, 2}-3}^{j}+\frac{1}{4} f_{k_{j, 2}-2}^{j}+\frac{1}{4} f_{k_{j, 2}-1}^{j}+\frac{1}{4} f_{k_{j, 2}}^{j}\right) \\
&=\frac{1}{4} \delta f_{k_{j, 2}-3}^{j}+\frac{1}{2} \delta f_{k_{j, 2}-2}^{j}+\frac{3}{4} \delta f_{k_{j, 2}-1}^{j}+\delta f_{k_{j, 2}}^{j}, \\
& \\
& \delta f_{2 k_{j, 2}-2}^{j+1}=0 \\
& \delta f_{2 k_{j, 2}-1}^{j+1}=\left(2 f_{k_{j, 2}+1}^{j}-f_{k_{j, 2}+2}^{j}\right)-f_{k_{j, 2}+1}^{j}, \\
&=-\delta f_{k_{j, 2}+1}^{j} .
\end{aligned}
$$

Since the difference (B.5) only depends on a difference involving points of $I_{2}, \delta f_{2 k_{j, 2}-1}^{j+1} \rightarrow_{j \rightarrow+\infty} 0$. Concerning $\delta f_{2 k_{j, 2}-3}^{j+1}$, the same argument as for Expression (B.4) is used since according to Definition $4, \forall j>J, k_{j, 2}=$ $2 k_{j-1,2}$. 


\section{References}

Baccou, J., Liandrat, J., 2005. Position-dependent lagrange interpolating multiresolutions. Int. J. of Wavelet, Multiresolution and Information 5(4), $513-539$.

Baccou, J., Liandrat, J., 2013. Kriging-based interpolatory subdivision schemes. Appl. Comput. Harmon. Anal. 35, 228-250.

Chiles, J., Delfiner, P., 1999. Geostatistics: Modeling spatial uncertainty. Wiley (New York).

Cohen, A., Dyn, N., Matei, B., 2003. Quasilinear subdivision schemes with applications to ENO interpolation. Appl. Comp. Harm. Anal. 15, 89-116.

Cressie, N., 1993. Statistics for spatial data. Wiley Series in Probability and Mathematical Statistics.

Deslauriers, G., Dubuc, S., 1987. Interpolation dyadique. In: Fractals, dimensions non entières et applications. Masson, Paris, pp. 44-45.

Durbin, J., Watson, G., 1951. Testing for serial correlation in least-squares regression. Biometrika 38, 159-171.

Dyn, N., 1992. Subdivision schemes in computer-aided geometric design. In: Light, W. (Ed.), Advances in Numerical analysis II, Wavelets, Subdivision algorithms and Radial Basis functions. Clarendon Press, Oxford, pp. 36104.

Dyn, N., Levin, D., 1995. Analysis of asymptotically equivalent binary subdivision schemes. J. Math. Anal. Appl. 193, 594-621.

Si, X., Baccou, J., Liandrat, J., 2014. Construction and analysis of zonedependent interpolatory/non-interpolatory stochastic subdivision schemes for non-regular data. Lecture Notes in Computer Science 8177, 456-470.

Wackernagel, H., 1998. Multivariate geostatistics. Springer. 


\section{List of Figures}

B.1 Coefficients of the stencil versus $j$ for the prediction of $f_{k}^{j}$ $(c=2)$. Left, $k=2 i$, Right, $k=2 i+1$. $\diamond$ stands for $\lambda_{0}^{(4)},+$ stands for $\lambda_{1}^{(4)}$ o stands for $\lambda_{2}^{(4)}$ and $*$ stands for $\lambda_{3}^{(4)}$. . . .

B.2 Behavior of the stencil coefficients with respect to $c$ for the prediction of $f_{k}^{j}(j=0)$. Left, $k=2 i$, right, $k=2 i+1{\text {. ' }{ }^{\prime}}^{\prime}$ stands for $\lambda_{0}^{(4)},{ }^{\prime} o^{\prime}$ stands for $\lambda_{1}^{(4)},{ }^{\prime}+{ }^{\prime}$ stands for $\lambda_{2}^{(4)}$ and ' $-^{\prime}$ stands for $\lambda_{3}^{(4)}$. . . . . . . . . . . . . . . . . . . . 38

B.3 Penalization function $\mathcal{P}$ for the 3-zone-dependent scheme . . . 39

B.4 Reconstruction of a step function; the circles stand for the sampled points, the solid line is the reconstructed function. $I_{1}$ is limited by the two straight lines. From top, left to bottom, right, $c=0$ (interpolatory Lagrange scheme), $c=10, c=30$ and $c=100$. . . . . . . . . . . . . . . . . . . . . 40

B.5 Reconstruction of a step function with a piecewise constant penalization functions defined by $c^{j}=c^{0}=30, I_{1}^{j}=I_{1}^{0}=$ $[5,10]$ (left) and $c^{j}=\frac{c^{0}}{2^{4 j}}, I_{1}^{j}=\left[8-\frac{3}{2^{j}}, 8+\frac{4}{2^{j}}\right]$ (right); the circles stand for the sampled points, the solid line is the reconstructed function. $I_{1}^{0}$ is limited by the two straight lines. . . . . . . . . 41

B.6 Reconstruction from noisy data with different values of $c$ (solid line); $*$ is associated to the initial data while the dashed line represents the original function. $I_{1}$ is limited by the two straight lines. From top to bottom and left to right, $c=0$, $c=10, c=100$ and $c=3726$.

B.7 Curve generation from a set of control points (cross signs), in which several ones are perturbated by a noise (circled cross signs). $I_{1}$ is limited by the two straight lines. Left, reconstruction by a Lagrange interpolatory scheme ; right, reconstruction by our penalized Lagrange subdivision with $c=100 \ldots$. . . . . 43

B.8 Prediction in the interior of $I_{1}$ : available values at level $j$ (o)

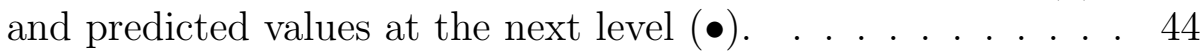

B.9 Prediction in the vicinity of $y_{1}=k_{j, 1} 2^{-j}$ : available values at level $j$ (o) and predicted values at the next level (•). . . . . . 45 

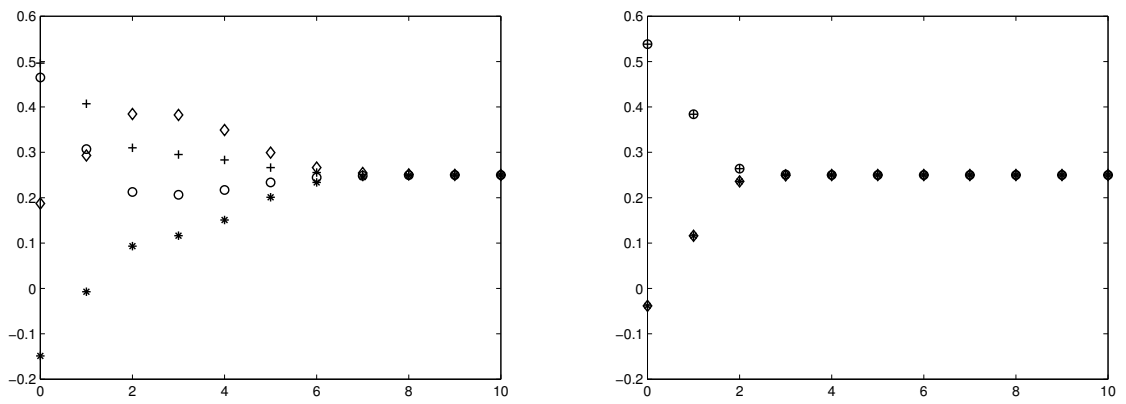

Figure B.1: Coefficients of the stencil versus $j$ for the prediction of $f_{k}^{j}(c=2)$. Left, $k=2 i$, Right, $k=2 i+1$. $\diamond$ stands for $\lambda_{0}^{(4)},+$ stands for $\lambda_{1}^{(4)}, o$ stands for $\lambda_{2}^{(4)}$ and $*$ stands for $\lambda_{3}^{(4)}$. 

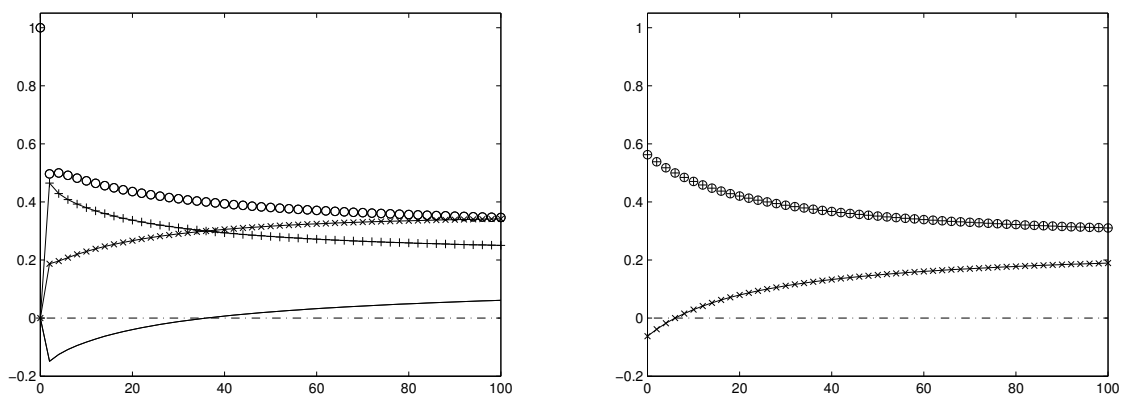

Figure B.2: Behavior of the stencil coefficients with respect to $c$ for the prediction of $f_{k}^{j}$ $(j=0)$. Left, $k=2 i$, right, $k=2 i+1 .{ }^{\prime} \times^{\prime}$ stands for $\lambda_{0}^{(4)},{ }^{\prime} o^{\prime}$ stands for $\lambda_{1}^{(4)},{ }^{\prime}+^{\prime}$ stands for $\lambda_{2}^{(4)}$ and ' $-^{\prime}$ stands for $\lambda_{3}^{(4)}$. 


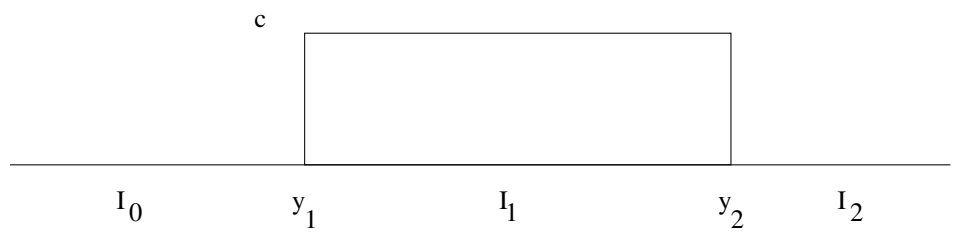

Figure B.3: Penalization function $\mathcal{P}$ for the 3-zone-dependent scheme 

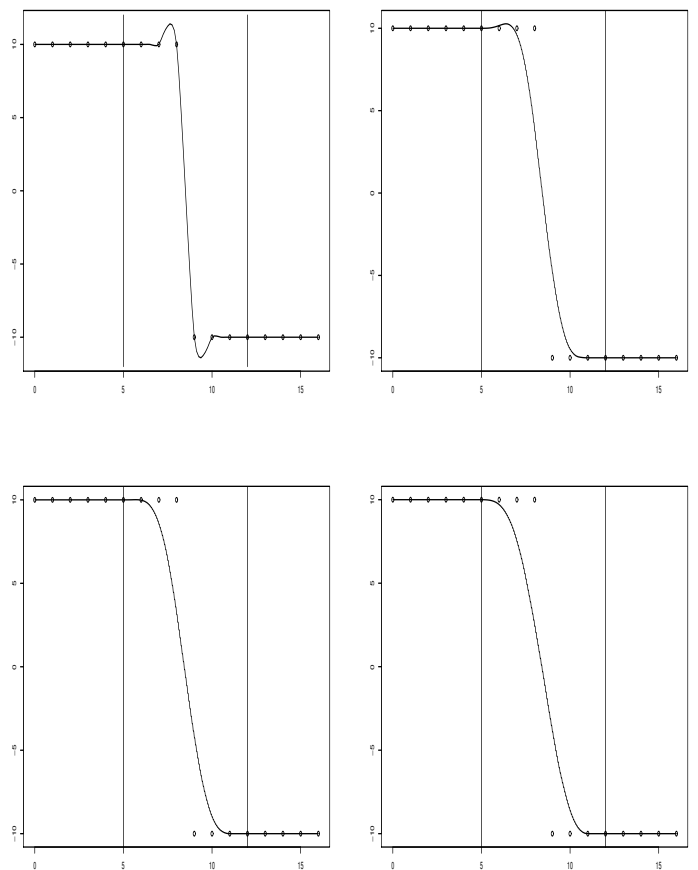

Figure B.4: Reconstruction of a step function; the circles stand for the sampled points, the solid line is the reconstructed function. $I_{1}$ is limited by the two straight lines. From top, left to bottom, right, $c=0$ (interpolatory Lagrange scheme), $c=10, c=30$ and $c=100$. 

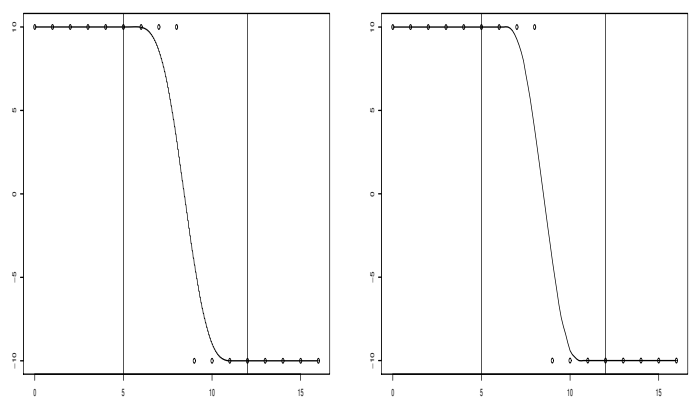

Figure B.5: Reconstruction of a step function with a piecewise constant penalization functions defined by $c^{j}=c^{0}=30, I_{1}^{j}=I_{1}^{0}=[5,10]$ (left) and $c^{j}=\frac{c^{0}}{2^{4 j}}, I_{1}^{j}=\left[8-\frac{3}{2^{j}}, 8+\frac{4}{2^{j}}\right]$ (right); the circles stand for the sampled points, the solid line is the reconstructed function. $I_{1}^{0}$ is limited by the two straight lines. . 

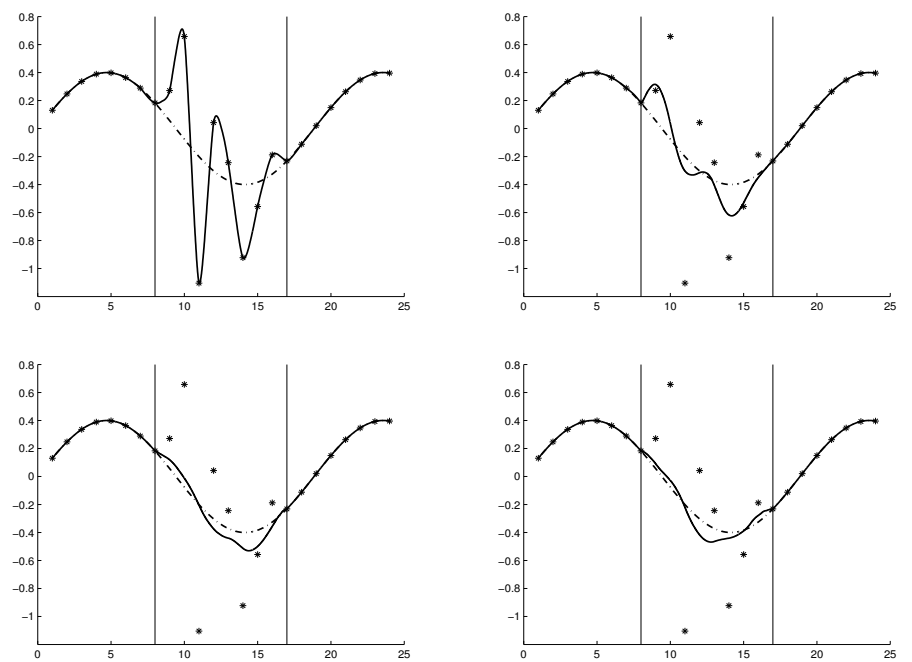

Figure B.6: Reconstruction from noisy data with different values of $c$ (solid line); $*$ is associated to the initial data while the dashed line represents the original function. $I_{1}$ is limited by the two straight lines. From top to bottom and left to right, $c=0, c=10$, $c=100$ and $c=3726$. 

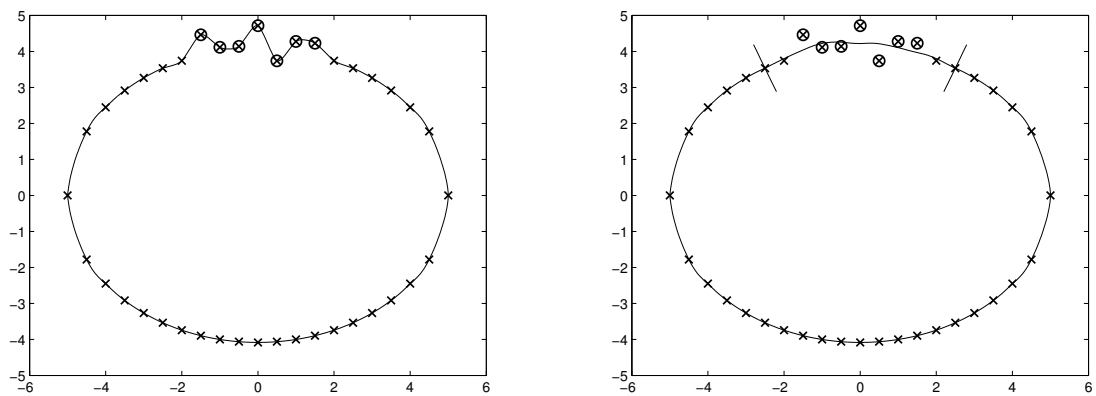

Figure B.7: Curve generation from a set of control points (cross signs), in which several ones are perturbated by a noise (circled cross signs). $I_{1}$ is limited by the two straight lines. Left, reconstruction by a Lagrange interpolatory scheme ; right, reconstruction by our penalized Lagrange subdivision with $c=100$. 


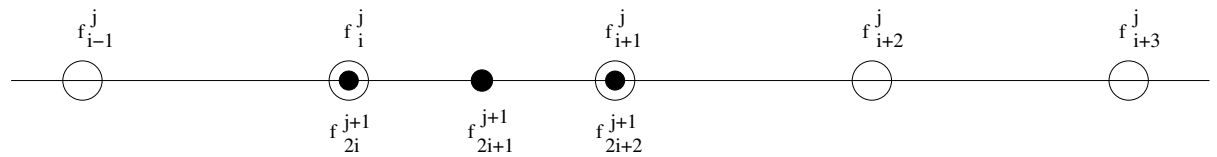

Figure B.8: Prediction in the interior of $I_{1}$ : available values at level $j$ (o) and predicted values at the next level $(\bullet)$. 


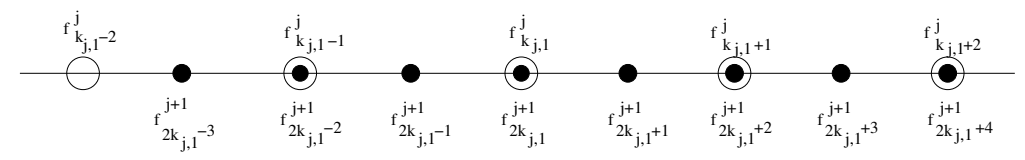

Figure B.9: Prediction in the vicinity of $y_{1}=k_{j, 1} 2^{-j}$ : available values at level $j$ (o) and predicted values at the next level $(\bullet)$. 


\section{List of Tables}

B.1 Limit stencils versus the penalization vector. . . . . . . . . 47

B.2 Critical values of $c$. The symbol "-" means that there is no positive value. . . . . . . . . . . . . . . . . . . . . . 48

B.3 Inferior bound of $c$ to reach the asymptotical stencil up to a $l_{2}$ error of $10^{-3}$. . . . . . . . . . . . . . . . . 49

B.4 Penalization vector and stencil associated to the zone-dependent (asymptotical) prediction. . . . . . . . . . . . 50

B.5 $l_{2}$-error between the sampling at level $j=6$ and the reconstructed sequence for different values of $c$. . . . . . . . . 51 


\begin{tabular}{|c|c|c|}
\hline Penalization vector & $k=2 i$ & $k=2 i+1$ \\
\hline$\left(0,0,0, c_{4}\right)$ & $(0,1,0,0)$ & $\left(L_{-2}^{2,1}\left(-\frac{1}{2}\right), L_{-1}^{2,1}\left(-\frac{1}{2}\right), L_{0}^{2,1}\left(-\frac{1}{2}\right), 0\right)$ \\
\hline$\left(0,0, c_{3}, c_{4}\right)$ & $(0,1,0,0)$ & $\left(L_{-2}^{2,0}\left(-\frac{1}{2}\right), L_{-1}^{2,0}\left(-\frac{1}{2}\right), 0,0\right)$ \\
\hline$\left(0, c_{2}, c_{3}, c_{4}\right)$ & $(1,0,0,0)$ & $(1,0,0,0)$ \\
\hline
\end{tabular}

Table B.1: Limit stencils versus the penalization vector. 


\begin{tabular}{|c|c|c|c|c|}
\hline $\mathrm{j}$ & $(c, 0,0,0)$ & $(c, c, 0,0)$ & $(c, c, c, 0)$ & $(c, c, c, c)$ \\
\hline 0 & 3 & 0.31 & - & 0.16 \\
\hline 1 & $4.5 e-2$ & $4.6 e-3$ & - & $2.3 e-3$ \\
\hline 2 & $7 e-4$ & $7 e-5$ & - & $3.5 e-5$ \\
\hline
\end{tabular}

Table B.2: Critical values of $c$. The symbol "-" means that there is no positive value. 


\begin{tabular}{|c|c|c|c|c|c|c|c|}
\hline & $\mathrm{j}=0$ & $\mathrm{j}=1$ & $\mathrm{j}=2$ & $\mathrm{j}=3$ & $\mathrm{j}=4$ & $\mathrm{j}=5$ & $\mathrm{j}=6$ \\
\hline $\mathrm{c}$ & 3726 & 232.9 & 14.5 & $9.1 e-1$ & $5.7 e-2$ & $3.5 e-3$ & $2.2 e-4$ \\
\hline
\end{tabular}

Table B.3: Inferior bound of $c$ to reach the asymptotical stencil up to a $l_{2}$-error of $10^{-3}$. 


\begin{tabular}{|c|c|c|c|}
\hline Position & Penalization vector & Stencil $(k=2 i)$ & Stencil $(k=2 i+1)$ \\
\hline $0 \leq i \leq k_{j, 1}-2$ & $(0,0,0,0)$ & $(0,1,0,0)$ & $\left(-\frac{1}{16}, \frac{9}{16}, \frac{9}{16},-\frac{1}{16}\right)$ \\
\hline$i=k_{j, 1}-1$ & $(0,0,0, \mathrm{c})$ & $(0,1,0,0)$ & $\left(-\frac{1}{8}, \frac{3}{4}, \frac{3}{8}, 0\right)$ \\
\hline$i=k_{j, 1}$ & $(0,0, \mathrm{c}, \mathrm{c})$ & $(0,1,0,0)$ & $\left(-\frac{1}{2}, \frac{3}{2}, 0,0\right)$ \\
\hline$i=k_{j, 1}+1$ & $(0, \mathrm{c}, \mathrm{c}, \mathrm{c})$ & $(1,0,0,0)$ & $(1,0,0,0)$ \\
\hline$k_{j, 1}+2 \leq i \leq k_{j, 2}-2$ & $(\mathrm{c}, \mathrm{c}, \mathrm{c}, \mathrm{c})$ & $\left(\frac{1}{4}, \frac{1}{4}, \frac{1}{4}, \frac{1}{4}\right)$ & $\left(\frac{1}{4}, \frac{1}{4}, \frac{1}{4}, \frac{1}{4}\right)$ \\
\hline$i=k_{j, 2}-1$ & $(\mathrm{c}, \mathrm{c}, \mathrm{c}, 0)$ & $(0,0,0,1)$ & $(0,0,0,1)$ \\
\hline$i=k_{j, 2}$ & $(\mathrm{c}, \mathrm{c}, 0,0)$ & $(0,0,2,-1)$ & $\left(0,0, \frac{3}{2},-\frac{1}{2}\right)$ \\
\hline$i=k_{j, 2}+1$ & $(\mathrm{c}, 0,0,0)$ & $(0,1,0,0)$ & $\left(0, \frac{3}{8}, \frac{3}{4},-\frac{1}{8}\right)$ \\
\hline$i \geq k_{j, 2}+2$ & $(0,0,0,0)$ & $(0,1,0,0)$ & $\left(-\frac{1}{16}, \frac{9}{16}, \frac{9}{16},-\frac{1}{16}\right)$ \\
\hline
\end{tabular}

Table B.4: Penalization vector and stencil associated to the zone-dependent (asymptotical) prediction. 


\begin{tabular}{|l|c|c|c|c|c|c|}
\hline & $c=0$ & $c=1$ & $c=10$ & $c=50$ & $c=100$ & $c=3726$ \\
\hline$l_{2}$-err & 5.6 & 2.9 & 2 & 1.2 & 1.05 & 0.85 \\
\hline
\end{tabular}

Table B.5: $l_{2}$-error between the sampling at level $j=6$ and the reconstructed sequence for different values of $c$. 